\title{
Gerstenhaber-Schack diagram cohomology from the operadic point of view
}

\author{
Martin Doubek
}

Received: 14 February 2011 / Accepted: 27 January 2012 / Published online: 13 March 2012

(C) Tbilisi Centre for Mathematical Sciences 2012

\begin{abstract}
We show that the operadic cohomology for any type of algebras over a non-symmetric operad $\mathcal{A}$ can be computed as Ext in the category of operadic $\mathcal{A}$-modules. We use this principle to prove that the Gerstenhaber-Schack diagram cohomology is operadic cohomology.
\end{abstract}

Keywords Homology · Operad

Mathematics Subject Classification (2000) $\quad 18 \mathrm{D} 50$

\section{Contents}

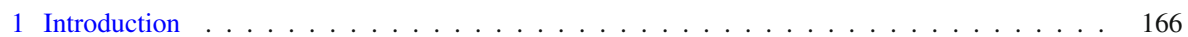

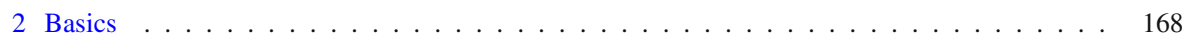

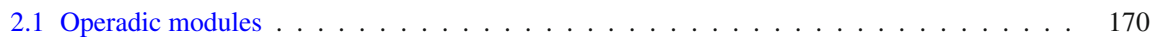

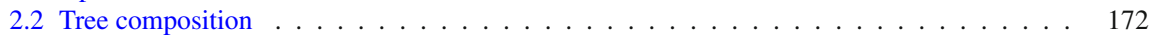

2.3 Free product of operads . . . . . . . . . . . . . . . . . . . . . . . . . . 174

3 Operadic cohomology of algebras . . . . . . . . . . . . . . . . . . . . 178

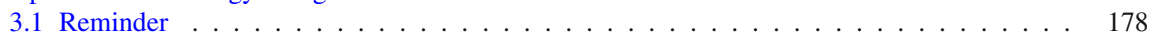

3.2 Algebras with derivation $\ldots \ldots \ldots \ldots \ldots$

3.3 Augmented cotangent complex . . . . . . . . . . . . . . . . . . . . . . . . . 188

4 Intermediate resolution of $\mathcal{D} \mathcal{A} \ldots \ldots \ldots \ldots \ldots \ldots \ldots$

4.1 From operads to operadic modules . . . . . . . . . . . . . . . . . . . 193

5 Gerstenhaber-Schack diagram cohomology is operadic cohomology . . . . . . . . . . . . . 196

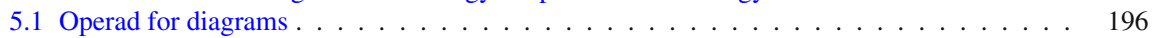

5.2 Gerstenhaber-Schack diagram cohomology . . . . . . . . . . . . . . . . . . . . . . . . . . 197

6 Resolution of $\mathcal{M D A} \ldots \ldots \ldots \ldots \ldots$. . . . . . . . . . . . . . . . . 198

Communicated by Jim Stasheff.

M. Doubek $(\bowtie)$

Mathematical Institute, Charles University, Sokolovská 83, 18675 Prague 8, Czech Republic e-mail: martindoubek@seznam.cz 


\section{Introduction}

The Operadic Cohomology (OC) gives a systematic way of constructing cohomology theories for algebras $A$ over an operad $\mathcal{A}$. It recovers the classical cases: Hochschild, Chevalley-Eilenberg, Harrison, etc. It also applies to algebras over coloured operads (e.g. morphism of algebras) and over PROPs (e.g. bialgebras). The OC first appeared in papers $[9,10]$ by Markl.

Abstractly, the $\mathrm{OC}$ is isomorphic to the triple cohomology, at least for algebras over Koszul operads [1]. It is also isomorphic to the André-Quillen Cohomology (AQC). In fact, the definition of OC is analogous to that of AQC: It computes the derived functor of the functor Der of derivations like AQC, but does so in the category of operads. While AQC offers a wider freedom for the choice of a resolution of the given algebra $A$, OC uses a particular universal resolution for all $\mathcal{A}$-algebras (this resolution is implicit, technically OC resolves the operad $\mathcal{A}$ ). Thus there is, for example, a universal construction of an $L_{\infty}$ structure on the complex computing OC [13], whose generalized Maurer-Cartan equation describes formal deformations of A.

The success of OC is due to the Koszul duality theory [7], which allows us to construct resolutions of Koszul operads explicitly. Koszul theory has received a lot of attention recently [19] and now goes beyond operads. However, it still has its limitations:

On one hand, it is bound to quadratic relations in a presentation of the operad $\mathcal{A}$. The problem with higher relations can be remedied by using a different presentation, but it comes at the cost of increasing the size of the resolution (e.g. [4]). This is not a major problem in applications, but the minimal resolutions have some nice properties - namely they are unique up to an isomorphism thus providing a cohomology theory unique already at the chain level. So the construction of the minimal resolutions is still of interest.

On the other hand, there are quadratic operads which are not Koszul and for those very little is known [15].

In this paper, we show that OC is isomorphic to Ext in the category of operadic $\mathcal{A}$-modules. Thus instead of resolving the operad $\mathcal{A}$, it suffices to find a projective resolution of a specific $\mathcal{A}$-module $\mathcal{M D} \mathcal{A}$ associated to $\mathcal{A}$. The ideas used here were already sketched in the paper [13] by Markl.

The resolution of operadic modules are probably much easier to construct explicitly than resolutions of operads, though this has to be explored yet. This simplification allows us to make a small step beyond Koszul theory:

An interesting example of a non-Koszul operad is the coloured operad describing a diagram of a fixed shape consisting of algebras over a fixed operad and morphisms of those algebras. The case of a single morphism between two algebras over a Koszul operad is long well understood. For a morphism between algebras over a general operad as well as for diagrams of a few simple shapes, some partial results were obtained in [12]. These are however not explicit enough to write down the OC.

On the other hand, a satisfactory cohomology for diagrams was invented by Gerstenhaber and Schack [5] as a natural simultaneous generalization of both Hochschild and simplicial cohomology. In the article [2], the authors proved that the 
Gerstenhaber-Schack cohomology of a single morphism of associative or Lie algebras is operadic cohomology. We use our theory to extend this result to arbitrary diagrams.

The method used can probably be applied in a more general context to show that a given cohomology theory is isomorphic to OC. The original example is [13] (and similar approach also appears in $[17,18]$ ), where the author proves that GerstenhaberSchack bialgebra cohomology is the operadic cohomology. Also the method might give an insight into the structure of operadic resolutions themselves, the problem we won't mention in this paper.

On the way, we obtain a modification of the usual OC which includes the quotient by infinitesimal automorphisms (Sect. 3.3).

Also an explicit description of a free resolution of the operad $\mathcal{A}$ with adjoined derivation is given if a free resolution of $\mathcal{A}$ is explicitly given. This appeared already in [13] and produces several new examples of minimal resolutions and as such might be of an independent interest.

We assume the reader is familiar with the language of operads (e.g. [8,16]).

Finally, I would like to thank Martin Markl for many useful discussions.

The author acknowledges the support by GAČR 201/09/H012 and also the hospitality of the Czech Academy of Sciences.

Convention 1.1 As our main object of interest is a diagram of associative algebras, we will get by with non-symmetric operads, that is operads with no action of the permutation groups. The results can probably be generalized in a straightforward way to symmetric operads.

In Sect. 2, we briefly recall basic notions of the operad theory with focus on coloured operads (see also [11,12]). We pay special attention to operadic modules. We introduce the notion of tree composition which is just a convenient way to write down complicated operadic compositions. In Sect. 2.3, we discuss free product of operads and obtain a form of the Künneth formula computing homology of the free product.

In Sect. 3, we develop the theory sketched by Markl in Appendix B of [13]. We give full details for coloured operads. We begin by recalling the operadic cohomology. In Sect. 3.2, we construct an explicit resolution of the operad $\mathcal{D} \mathcal{A}$ describing algebras over $\mathcal{A}$ with adjoined derivation assuming we know an explicit resolution of the operad $\mathcal{A}$. In Sect. 3.3, we clarify the significance of operadic derivations on the resolution of $\mathcal{D} \mathcal{A}$ with values in $\mathcal{E} n d_{A}$. This leads to an augmentation of the cotangent complex which has nice interpretation in terms of formal deformation theory. In Sects. 4 and 4.1 , we realize that all the information needed to construct augmented cohomology is contained in a certain operadic module. This module is intrinsically characterized by being a resolution (in the category of operadic modules) of $\mathcal{M D} \mathcal{A}$, a certain module constructed from $\mathcal{A}$ in a very simple way.

In Sect. 5, we apply the theory to prove that the Gerstenhaber-Schack diagram cohomology is isomorphic to the operadic cohomology. We begin by explaining how a diagram of associative algebras is described by an operad $\mathcal{A}$. We also make the associated module $\mathcal{M D} \mathcal{A}$ explicit. Then we recall the Gerstenhaber-Schack cohomology and obtain a candidate for a resolution of $\mathcal{M D} \mathcal{A}$. In Sect. 6, we verify that the candidate is a valid resolution. This computation is complicated, but still demonstrates the technical advantage of passing to the modules. 


\section{Basics}

Fix the following symbols:

- $C$ is a set of colours.

- $k$ is a field of characteristics 0 .

- $\mathbb{N}_{0}$ is the set of natural numbers including 0 .

We will also use the following notations and conventions:

- Vector spaces over $k$ are called $k$-modules, chain complexes of vector spaces over $k$ with differential of degree -1 are called $d g-k$-modules and morphisms of chain complexes are called just maps. Chain complexes are assumed non-negatively graded unless stated otherwise.

- $|x|$ is the degree of an element $x$ of a dg- $k$-module.

- $H_{*}(A)$ is homology of the object $A$, whatever $A$ is.

- $k\langle S\rangle$ is the $k$-linear span of the set $S$.

- $\operatorname{ar}(v)$ is arity of the object $v$, whatever $v$ is.

- Quism is a map $f$ of dg- $k$-modules such that the induced map $H_{*}(f)$ on homology is an isomorphism.

Definition 2.1 A dg- $C$-collection $X$ is a set

$$
\left\{X\left(\begin{array}{c}
c \\
c_{1}, \ldots, c_{n}
\end{array}\right) \mid n \in \mathbb{N}_{0}, c, c_{1}, \ldots, c_{n} \in C\right\}
$$

of dg- $k$-modules. We call $c$ the output colour of elements of $X\left(\begin{array}{c}c \\ c_{1}, \ldots, c_{n}\end{array}\right), c_{1}, \ldots, c_{n}$ are the input colours, $n$ is the arity. We also admit $n=0$.

When the above dg- $k$-modules have zero differentials, we talk just about graded $C$-collection. If moreover no grading is given, we talk just about $C$-collection. All notions that follow have similar analogues. If the context is clear, we might omit the prefixes dg- $C$ completely.

A dg- $C$-operad $\mathcal{A}$ is a dg- $C$-collection $\mathcal{A}$ together with a set of dg-k-module maps

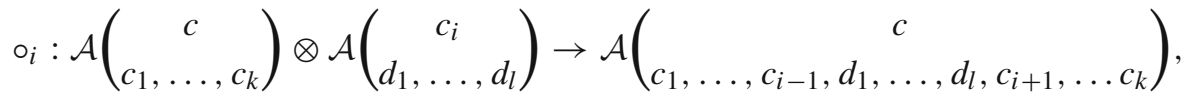

called operadic compositions, one for each choice of $k, l \in \mathbb{N}_{0}, 1 \leqslant i \leqslant k$ and $c, c_{1}, \ldots, c_{k}, d_{1}, \ldots, d_{l} \in C$, and a set of units

$$
e: k \rightarrow \mathcal{A}\left(\begin{array}{l}
c \\
c
\end{array}\right)
$$

one for each $c \in C$. These maps satisfy the usual associativity and unit axioms, e.g. [16].

The initial dg- $C$-operad is denoted $I$. 
Equivalently, $\mathrm{dg}$ - $C$-operad is a monoid in the monoidal category of $\mathrm{dg}$ - $C$-collections with the composition product $\circ$ :

$$
\begin{aligned}
& (\mathcal{A} \circ \mathcal{B})\left(\begin{array}{c}
c \\
c_{1}, \ldots, c_{n}
\end{array}\right) \\
& :=\bigoplus_{\substack{k \geqslant 0, i_{1}, \ldots, i_{k} \geqslant 0, d_{1}, \ldots, d_{k} \in C}} \mathcal{A}\left(\begin{array}{c}
c \\
d_{1}, \ldots, d_{k}
\end{array}\right) \otimes \mathcal{B}\left(\begin{array}{c}
d_{1} \\
c_{1}, \ldots, c_{i_{1}}
\end{array}\right) \otimes \cdots \otimes \mathcal{B}\left(\begin{array}{c}
d_{k} \\
c_{i_{1}+\cdots i_{k-1}+1}, \ldots, c_{n}
\end{array}\right) .
\end{aligned}
$$

In contrast to the uncoloured operads, the composition is defined only for the "correct" colours and there is one unit in every colour, i.e. $I\left(\begin{array}{l}c \\ c\end{array}\right)=k$ for every $c \in C$. Hence we usually talk about the units. We denote by $1_{c}$ the image of $1 \in k=I\left(\begin{array}{l}c \\ c\end{array}\right)$, hence $\operatorname{Im} e=\bigoplus_{c \in C} k\left\langle 1_{c}\right\rangle$. The notation $1_{c}$ for units coincides with the notation for identity morphisms. The right meaning will always be clear from the context.

For a dg-C-operad $\mathcal{A}$, we can consider its homology $H_{*}(\mathcal{A})$. The operadic composition descends to $H_{*}(\mathcal{A})$. Obviously, the units $1_{c}$ are concentrated in degree 0 and by our convention on non-negativity of the grading, $1_{c}$ defines a homology class $\left[1_{c}\right]$. It is a unit in $H^{*}(\mathcal{A})$. It can happen that $\left[1_{c}\right]=0$ in which case it is easily seen that $H_{*}(\mathcal{A})\left(\begin{array}{c}c_{0} \\ c_{1}, \ldots, c_{n}\end{array}\right)=0$ whenever any of $c_{i}$ 's equals $c$. If all [ $1_{c}$ ]'s are nonzero, then $H_{*}(\mathcal{A})$ is a graded $C$-operad.

Let $\mathcal{M}_{1}$ and $\mathcal{M}_{2}$ be two dg-C-collections. Then $\mathbf{d g}$ - $C$-collection morphism $f$ is a set of dg maps

$$
f\left(\begin{array}{c}
c \\
c_{1}, \ldots, c_{n}
\end{array}\right): \mathcal{M}_{1}\left(\begin{array}{c}
c \\
c_{1}, \ldots, c_{n}
\end{array}\right) \rightarrow \mathcal{M}_{2}\left(\begin{array}{c}
c \\
c_{1}, \ldots, c_{n}
\end{array}\right),
$$

one for each $n \in \mathbb{N}_{0}, c, c_{1}, \ldots, c_{n} \in C$.

The dg- $C$-collection morphisms are composed "colourwise" in the obvious way.

A dg- $C$-operad morphism is a dg- $C$-collection morphisms preserving the operadic compositions and units.

Recall that given a dg- $k$-module $A$, the endomorphism operad $\mathcal{E} n d_{A}$ is equipped with the differential

$$
\partial_{\mathcal{E}_{n d_{A}}} f:=\partial_{A} f-(-1)^{|f|} f \partial_{A^{\otimes n}}
$$

for $f \in \mathcal{E} n d_{A}(n)$ homogeneous. Let there be a decomposition

$$
\left(A, \partial_{A}\right)=\bigoplus_{c \in C}\left(A_{c}, \partial_{A_{c}}\right)
$$

Then the endomorphism operad is naturally a $\mathrm{dg}-C$-operad via

$$
\mathcal{E}_{n d}\left(\begin{array}{c}
c \\
c_{1}, \ldots, c_{n}
\end{array}\right):=\operatorname{Hom}_{k}\left(A_{c_{1}} \otimes \cdots \otimes A_{c_{n}}, A_{c}\right) .
$$


An algebra over a dg- $C$-operad $\mathcal{A}$ is a dg- $C$-operad morphism

$$
\left(\mathcal{A}, \partial_{\mathcal{A}}\right) \rightarrow\left(\mathcal{E}_{n d_{A}}, \partial_{\mathcal{E}_{n d_{A}}}\right)
$$

\subsection{Operadic modules}

Definition 2.2 Let $\mathcal{A}=\left(\mathcal{A}, \partial_{\mathcal{A}}\right)$ be a dg-C-operad. An (operadic) $\mathbf{d g}$ - $\mathcal{A}$-module $\mathcal{M}$ is a dg-C-collection

$$
\left\{\mathcal{M}\left(\begin{array}{c}
c \\
c_{1}, \ldots, c_{n}
\end{array}\right) \mid n \in \mathbb{N}_{0}, c, c_{1}, \ldots, c_{n} \in C\right\}
$$

with structure maps

$$
\begin{aligned}
\mathrm{o}_{i}^{L}: \mathcal{A}\left(\begin{array}{c}
c \\
c_{1}, \ldots, c_{k}
\end{array}\right) \otimes \mathcal{M}\left(\begin{array}{c}
c_{i} \\
d_{1}, \ldots, d_{l}
\end{array}\right) \rightarrow \mathcal{M}\left(\begin{array}{c}
c \\
c_{1}, \ldots, c_{i-1}, d_{1}, \ldots, d_{l}, c_{i+1}, \ldots c_{k}
\end{array}\right), \\
\circ_{i}^{R}: \mathcal{M}\left(\begin{array}{c}
c \\
c_{1}, \ldots, c_{k}
\end{array}\right) \otimes \mathcal{A}\left(\begin{array}{c}
c_{i} \\
d_{1}, \ldots, d_{l}
\end{array}\right) \rightarrow \mathcal{M}\left(\begin{array}{c}
c \\
c_{1}, \ldots, c_{i-1}, d_{1}, \ldots, d_{l}, c_{i+1}, \ldots c_{k}
\end{array}\right),
\end{aligned}
$$

one for each choice of $c, c_{1}, \ldots, d_{1}, \ldots \in C$ and $1 \leqslant i \leqslant k$. These structure maps are required to satisfy the expected axioms:

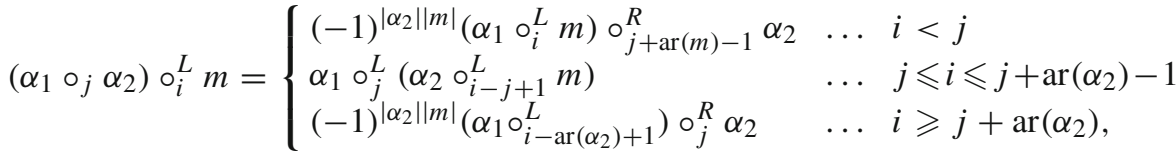

$$
\begin{aligned}
& m \circ_{i}^{R}\left(\alpha_{1} \circ_{j} \alpha_{2}\right)=\left(m \circ_{i}^{R} \alpha_{1}\right) \circ_{j+i-1}^{R} \alpha_{2} \text {, }
\end{aligned}
$$

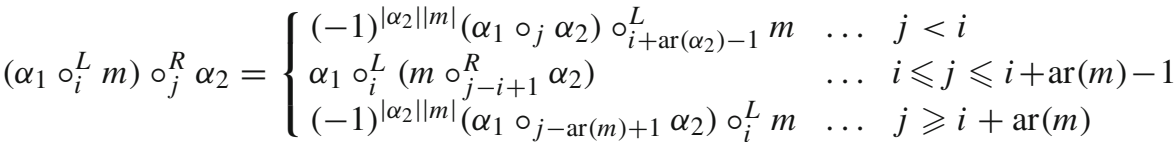

and

$$
1_{c} \circ_{1} m=m=m \circ_{i} 1_{c_{i}} \quad 1 \leqslant i \leqslant \operatorname{ar}(m)
$$

for $\alpha_{1}, \alpha_{2} \in \mathcal{A}$ and $m \in \mathcal{M}\left(\begin{array}{c}c \\ c_{1}, \ldots, c_{\operatorname{ar}(m)}\end{array}\right)$ in the correct colours. We usually omit the upper indices $L, R$, writing only $\circ_{i}$ for all the operations.

A morphism of dg- $\mathcal{A}$-modules $\mathcal{M}_{1}, \mathcal{M}_{2}$ is a dg-C-collection morphism $\mathcal{M}_{1} \stackrel{f}{\rightarrow}$ $\mathcal{M}_{2}$ satisfying

$$
\begin{aligned}
& f\left(a \circ \circ_{i}^{L} m\right)=a \circ_{i}^{L} f(m), \\
& f\left(m \circ \circ_{i}^{R} a\right)=f(m) \circ{ }_{i}^{R} a .
\end{aligned}
$$


We expand the definition of dg- $\mathcal{A}$-module. Recall that each $\mathcal{M}\left({ }_{c_{1}, \ldots, c_{n}}{ }^{c}\right)$ is a dg-kmodule. The differentials of these $\mathrm{dg}-k$-modules define a $\mathrm{dg}-C$-collection morphism $\partial_{\mathcal{M}}: \mathcal{M} \rightarrow \mathcal{M}$ of degree -1 satisfying $\partial_{\mathcal{M}}^{2}=0$. The structure maps $\circ_{i}^{L}$ and $\circ_{i}^{R}$ commute with the differentials on the tensor products. Hence $\partial_{\mathcal{M}}: \mathcal{M} \rightarrow \mathcal{M}$ is a derivation in the following sense:

$$
\begin{aligned}
& \partial_{\mathcal{M}}\left(a \circ_{i} m\right)=\partial_{\mathcal{A}} a \circ_{i} m+(-1)^{|a|} a \circ_{i} \partial_{\mathcal{M}} m \\
& \partial_{\mathcal{M}}\left(m \circ_{i} a\right)=\partial_{\mathcal{M}} m \circ_{i} a+(-1)^{|m|} m \circ_{i} \partial_{\mathcal{A}} a
\end{aligned}
$$

As in the case of modules over a ring, $\mathcal{A}$-modules form an abelian category. We have "colourwise" kernels, cokernels, submodules, etc. There is a free $\mathcal{A}$-module generated by a $C$-collection $M$, denoted

$$
\mathcal{A}\langle M\rangle
$$

and satisfying the usual universal property. As an example of an explicit description of $\mathcal{A}\langle M\rangle$, let $\mathcal{A}:=\mathbb{F}\left(M_{1}\right)$ be a free $C$-operad generated by a $C$-collection $M_{1}$. Then $\mathcal{A}\left\langle M_{2}\right\rangle$ is spanned by all planar trees, whose exactly one vertex is decorated by an element of $M_{2}$ and all the other vertices are decorated by elements of $M_{1}$ such that the colours are respected in the obvious sense.

We warn the reader that the notion of operadic module varies in the literature. For example the monograph [3] uses a different definition.

While dealing with $\mathcal{A}$-modules, it is useful to introduce the following infinitesimal composition product $\mathcal{A} \circ^{\prime}(\mathcal{B}, \mathcal{C})$ of $C$-collections $\mathcal{A}, \mathcal{B}, \mathcal{C}$ :

$$
\begin{aligned}
& \left(\mathcal{A} \circ \circ^{\prime}(\mathcal{B}, \mathcal{C})\right)\left(\begin{array}{c}
c \\
c_{1}, \ldots, c_{n}
\end{array}\right):=\underbrace{}_{\substack{k \geqslant 0, l>0, 0 \leqslant i_{1} \leqslant \ldots \leqslant i_{k-1} \leqslant n, d_{1}, \ldots, d_{k} \in C}} \mathcal{A}\left(\begin{array}{c}
c \\
d_{1}, \ldots, d_{k}
\end{array}\right) \otimes \\
& \otimes \mathcal{B}\left(\begin{array}{c}
d_{1} \\
c_{1}, \ldots, c_{i_{1}}
\end{array}\right) \otimes \cdots \otimes \underbrace{\mathcal{C}\left(\begin{array}{c}
d_{l} \\
c_{i_{l-1}+1}, \ldots, c_{i_{l}}
\end{array}\right)}_{\text {lth-position }} \otimes \cdots \otimes \mathcal{B}\left(\begin{array}{c}
d_{k} \\
c_{i_{k-1}+1}, \ldots, c_{n}
\end{array}\right) .
\end{aligned}
$$

See also [8]. We denote by

$$
\mathcal{A} \circ_{l}^{\prime}(\mathcal{B}, \mathcal{C})
$$

the projection of $\mathcal{A} \circ^{\prime}(\mathcal{B}, \mathcal{C})$ onto the component with fixed $l$.

For the free module, we have the following description using the infinitesimal composition product:

$$
\mathcal{A}\langle M\rangle \cong \mathcal{A} \circ^{\prime}(I, M \circ \mathcal{A}) .
$$




\subsection{Tree composition}

An (unoriented) graph (without loops) is a set $V$ of vertices, a set $H_{v}$ of half edges for every $v \in V$ and a set $E$ of (distinct) unordered pairs (called edges) of distinct elements of $V$. If $e:=(v, w) \in E$, we say that the vertices $v, w$ are adjacent to the edge $e$ and the edge $e$ is adjacent to the vertices $v, w$. Denote $E_{v}$ the set of all edges adjacent to $v$. Similarly, for $h \in H_{v}$, we say that the vertex $v$ is adjacent to the half edge $h$ and vice versa. A path connecting vertices $v, w$ is a sequence $\left(v, v_{1}\right),\left(v_{1}, v_{2}\right), \ldots,\left(v_{n}, w\right)$ of distinct edges. A tree is a graph such that for every two vertices $v, w$ there is a path connecting them iff $v \neq w$. A rooted tree is a tree with a chosen half edge, called root. The root vertex is the unique vertex adjacent to the root. The half edges other than the root are called leaves or legs. For every vertex $v$ except for the root vertex, there is a unique edge $e_{v} \in E_{v}$ contained in the unique path connecting $v$ to the root vertex. The edge $e_{v}$ is called output and the other edges and half edges adjacent to $v$ are called legs or inputs of $v$. The root is, by definition, the output of the root vertex. The number of legs of $v$ is called arity of $v$ and is denoted $\operatorname{ar}(v)$. Notice we also admit vertices with no legs, i.e. vertices of arity 0 . A planar tree is a tree with a given ordering of the set $H_{v} \sqcup E_{v}-\left\{e_{v}\right\}$ for each $v \in V$ (the notation $\sqcup$ stands for the disjoint union). The planarity induces an ordering on the set of all leaves, e.g. by numbering them $1,2, \ldots$. For example,

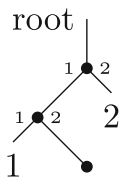

is a planar tree with 3 vertices, 3 half edges and 2 edges. We use the convention that the topmost half edge is always the root. Then there are 2 leaves. The planar ordering of legs of all vertices is denoted by small numbers and the induced ordering of leaves is denoted by big numbers.

Let $T_{1}, T_{2}$ be two planar rooted trees, let $T_{1}$ have $n$ leaves and for $j=1,2$ let $V_{j}$ resp. $H_{j, v}$ resp. $E_{j}$ denote the set of vertices resp. half edges resp. edges of $T_{j}$. For $1 \leqslant i \leqslant n$ we have the grafting operation $\circ_{i}$ producing a planar rooted tree $T_{1} \circ_{i} T_{2}$ defined as follows: first denote $l$ the $i$ th leg of $T_{1}$ and denote $v_{1}$ the vertex adjacent to $l$, denote $r$ the root of $T_{2}$ and denote $v_{2}$ the root vertex of $T_{2}$. Then the set of vertices of $T_{1} \circ_{i} T_{2}$ is $V_{1} \sqcup V_{2}$, the set $H_{v}$ of half edges is

$$
H_{v}=\left\{\begin{array}{lll}
H_{1, v} & \ldots & v \in V_{1}-\left\{v_{1}\right\} \\
H_{1, v_{1}}-\{l\} & \ldots & v=v_{1} \\
H_{2, v} & & v \in V_{2}-\left\{v_{2}\right\} \\
H_{2, v_{2}}-\{r\} & \ldots & v=v_{2}
\end{array}\right.
$$

and finally the set of edges is $E_{1} \sqcup E_{2} \sqcup\left\{\left(v_{1}, v_{2}\right)\right\}$. The planar structure is inherited in the obvious way. For example, 


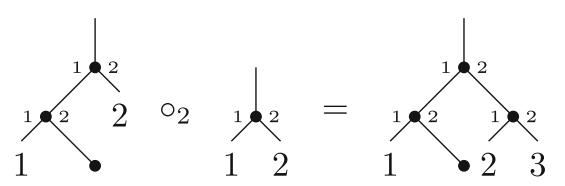

From this point on, tree will always mean a planar rooted tree. Such trees can be used to encode compositions of elements of an operad including those of arity 0.

Let $T$ be a tree with $n$ vertices $v_{1}, \ldots, v_{n}$. Suppose moreover that the vertices of $T$ are ordered, i.e. there is a bijection $b:\left\{v_{1}, \ldots, v_{n}\right\} \rightarrow\{1, \ldots, n\}$. We denote such a tree with ordered vertices by $T_{b}$.

Now we explain how the bijection $b$ induces a structure of tree with levels on $T_{b}$ such that each vertex is on a different level. Intuitively, $b$ encodes in what order are elements of an operad composed. We formalize this as follows:

Let $p_{1}, \ldots, p_{n} \in \mathcal{P}$ be elements of a dg- $C$-operad $\mathcal{P}$ such that if two vertices $v_{i}, v_{j}$ are adjacent to a common edge $e$, which is simultaneously the $l$ th leg of $v_{i}$ and the output of $v_{j}$, then the $l$ th input colour of $p_{i}$ equals the output colour of $p_{j}$. We say that $v_{i}$ is decorated by $p_{i}$. Define inductively: Let $i$ be such that $v_{i}$ is the root vertex. Define

$$
\begin{aligned}
T^{1} & :=v_{i}, \\
T_{b}^{1}\left(p_{1}, \ldots, p_{n}\right) & :=p_{i} .
\end{aligned}
$$

Here we are identifying $v_{i}$ with the corresponding corolla. Assume a subtree $T^{k-1}$ of $T$ and $T_{b}^{k-1}\left(p_{1}, \ldots, p_{n}\right) \in \mathcal{P}$ are already defined. Consider the set $J$ of all $j$ 's such that $v_{j} \notin T^{k-1}$ and there is an edge $e$ between $v_{j}$ and some vertex $v$ in $T^{k-1}$. Let $i \in J$ be such that $b\left(v_{i}\right)=\min \left\{b\left(v_{j}\right): j \in J\right\}$. Let $l$ be the number of the leg $e$ of vertex $v$ in the planar ordering of $T$ and define

$$
\begin{aligned}
T^{k} & :=T^{k-1} \circ_{l} v_{i}, \\
T_{b}^{k}\left(p_{1}, \ldots, p_{n}\right) & :=T_{b}^{k-1}\left(p_{1}, \ldots, p_{n}\right) \circ p_{i} .
\end{aligned}
$$

In the upper equation, we are using the operation ${ }_{l}$ of grafting of trees. Finally

$$
T_{b}\left(p_{1}, \ldots, p_{n}\right):=T_{b}^{n}\left(p_{1}, \ldots, p_{n}\right) .
$$

$T_{b}\left(p_{1}, \ldots, p_{n}\right)$ is called tree composition of $p_{1}, \ldots, p_{n}$ along $T_{b}$.

If $T$ and $p_{i}$ 's are fixed, changing $b$ may change the sign of $T_{b}\left(p_{1}, \ldots, p_{n}\right)$. Observe that if $\mathcal{P}$ is concentrated in even degrees (in particular 0 ) then the sign doesn't change. If $b$ is understood and fixed, we usually omit it.

For example, let

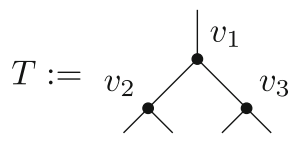


For $i=1,2,3$, let $p_{i}$ be an element of degree 1 and arity 2 . Let $b\left(v_{1}\right)=1, b\left(v_{2}\right)=$ $2, b\left(v_{3}\right)=3$ and $b^{\prime}\left(v_{1}\right)=1, b^{\prime}\left(v_{2}\right)=3, b\left(v_{3}\right)=2$. Then

$$
T_{b}\left(p_{1}, p_{2}, p_{3}\right)=\left(p_{1} \circ_{1} p_{2}\right) \circ_{3} p_{3} \quad \text { and } \quad T_{b^{\prime}}\left(p_{1}, p_{2}, p_{3}\right)=\left(p_{1} \circ_{2} p_{3}\right) \circ_{1} p_{2}
$$

and by the associativity axiom

$$
T_{b}\left(p_{1}, p_{2}, p_{3}\right)=-T_{b^{\prime}}\left(p_{1}, p_{2}, p_{3}\right) .
$$

A useful observation is that we can always reindex $p_{i}$ 's so that

$$
T_{b}\left(p_{1}, \ldots, p_{n}\right)=\left(\cdots\left(\left(p_{1} \circ_{i_{1}} p_{2}\right) \circ_{i_{2}} p_{3}\right) \cdots \circ_{i_{n-1}} p_{n}\right)
$$

for some $i_{1}, i_{2}, \ldots, i_{n-1}$.

Tree compositions are a convenient notation for dealing with operadic derivations.

\subsection{Free product of operads}

Definition 2.3 Free product $\mathcal{A} * \mathcal{B}$ of $\operatorname{dg}$ - $C$-operads $\mathcal{A}, \mathcal{B}$ is the coproduct $\mathcal{A} \amalg \mathcal{B}$ in the category of $\mathrm{dg}$ - $C$-operads.

Let $A, B$ be dg- $k$-modules. The usual Künneth formula states that the map

$$
\begin{gathered}
H_{*}(A) \otimes H_{*}(B) \stackrel{\iota}{\rightarrow} H_{*}(A \otimes B) \\
{[a] \otimes[b] \mapsto[a \otimes b]}
\end{gathered}
$$

is a natural isomorphism of dg-k-modules, where [ ] denotes a homology class. Our aim here is to prove an analogue of the Künneth formula for the free product of operads, that is

$$
H_{*}(\mathcal{A}) * H_{*}(\mathcal{B}) \cong H_{*}(\mathcal{A} * \mathcal{B})
$$

naturally as $C$-operads.

First we describe $\mathcal{A} * \mathcal{B}$ more explicitly. Intuitively, $\mathcal{A} * \mathcal{B}$ is spanned by trees whose vertices are decorated by elements of $\mathcal{A}$ or $\mathcal{B}$ such that no two vertices adjacent to a common edge are both decorated by $\mathcal{A}$ or both by $\mathcal{B}$. Unfortunately, this is not quite true-there are problems with units of the operads.

Recall a dg- $C$-operad $\mathcal{P}$ is called augmented iff there is a dg- $C$-operad morphism $\mathcal{P} \stackrel{a}{\rightarrow} I$ inverting the unit of $\mathcal{P}$ on the left, i.e. the composition $I \stackrel{e}{\rightarrow} \mathcal{P} \stackrel{a}{\rightarrow} I$ is $1_{I}$. The kernel of $a$ is denoted by $\overline{\mathcal{P}}$ and usually called augmentation ideal.

If $\mathcal{A}, \mathcal{B}$ are augmented, we let the vertices be decorated by the augmentation ideals $\overline{\mathcal{A}}, \overline{\mathcal{B}}$ instead of $\mathcal{A}, \mathcal{B}$ and the above description of $\mathcal{A} * \mathcal{B}$ works well. In fact, this has been already treated in [11]. 
However we will work without the augmentation assumption. Choose a sub- $C$ collection $\overline{\mathcal{A}}$ of $\mathcal{A}$ such that

$$
\begin{gathered}
\overline{\mathcal{A}} \oplus 1_{\mathcal{A}}=\mathcal{A}, \\
\operatorname{Im} \partial_{\mathcal{A}} \subset \overline{\mathcal{A}},
\end{gathered}
$$

where

$$
1_{\mathcal{A}}:=\bigoplus_{c \in C} k\left\langle 1_{c}\right\rangle
$$

This is possible iff

$$
\left[1_{c}\right] \neq 0 \text { for all } c \in C \text {, }
$$

that is if $H_{*}(\mathcal{A})$ is a graded $C$-operad. This might not be the case generally as we have already seen at the beginning of Sect. 2 so let's assume it. Choose $\overline{\mathcal{B}}$ for $\mathcal{B}$ similarly.

For given $\overline{\mathcal{A}}, \overline{\mathcal{B}}$, a free product tree is a tree $T$ together with

$$
c(v), c_{1}(v), c_{2}(v), \ldots, c_{\operatorname{ar}(v)}(v) \in C \text { for each vertex } v
$$

and a map

$$
\overline{\mathcal{P}}: \text { vertices of } T \rightarrow\{\overline{\mathcal{A}}, \overline{\mathcal{B}}\}
$$

such that if vertices $v_{1}, v_{2}$ are adjacent to a common edge, which is simultaneously the $l$ th leg of $v_{1}$ and the output of $v_{2}$, then

$$
c_{l}\left(v_{1}\right)=c\left(v_{2}\right) \quad \text { and } \quad \overline{\mathcal{P}}\left(v_{1}\right) \neq \overline{\mathcal{P}}\left(v_{2}\right) .
$$

Finally, the description of the free product is as follows:

$$
\mathcal{A} * \mathcal{B}:=\bigoplus_{c \in C} k\left\langle 1_{c}\right\rangle \oplus \bigoplus_{T} \bigotimes_{v} \overline{\mathcal{P}}(v)\left(\begin{array}{c}
c(v) \\
c_{1}(v), \ldots, c_{\operatorname{ar}(v)}(v)
\end{array}\right)
$$

where $T$ runs over all isomorphism classes of free product trees and $v$ runs over all vertices of $T$ and $1_{c}$ 's are of degree 0 . If the vertices of $T$ are $v_{1}, \ldots, v_{n}$ then every element of $\bigotimes_{v} \overline{\mathcal{P}}(v)\left(_{c_{1}(v), \ldots, c_{\operatorname{ar}(v)}(v)}^{c(v)}\right)$ can be written as a tree composition $T\left(x_{1}, \ldots, x_{n}\right)$ where $x_{i} \in \overline{\mathcal{P}}\left(v_{i}\right)$. Hence $v_{i}$ is decorated by $x_{i}$.

The operadic composition

$$
T\left(x_{1}, \ldots, x_{n}\right) \circ_{i} T^{\prime}\left(x_{1}^{\prime}, \ldots, x_{m}^{\prime}\right)
$$


in $\mathcal{A} * \mathcal{B}$ is defined in the obvious way by grafting $T$ and $T^{\prime}$ (the result $T \circ_{i} T^{\prime}$ of the grafting may not be a free product tree) and then (repeatedly) applying the following reducing operations:

1. Suppose $w_{1}, w_{2}$ are vertices of $T \circ_{i} T^{\prime}$ adjacent to a common edge $e$ which is simultaneously the $l$ th leg of $w_{1}$ and the output of $w_{2}$. Suppose moreover that $w_{1}$ is decorated by $p_{1}$ and $w_{2}$ by $p_{2}$. If both $p_{1}, p_{2}$ are elements of $\overline{\mathcal{A}}$ or both of $\overline{\mathcal{B}}$, then contract $e$ and decorate the resulting vertex by the composition of $p_{1} \circ_{l} p_{2}$.

2. If a vertex is decorated by a unit from $1_{\mathcal{A}}$ or $1_{\mathcal{B}}$ (this may happen since neither $\overline{\mathcal{A}}$ nor $\overline{\mathcal{B}}$ is generally closed under the composition!), omit it unless it is the only remaining vertex of the tree.

After several applications of the above reducing operations, we obtain a free product tree or a tree with a single vertex decorated by a unit.

Obviously, $1_{c}$ 's are units for this composition.

The differential $\partial$ on $\mathcal{A} * \mathcal{B}$ is determined by (8) and the requirement that $\partial\left(1_{c}\right)=0$ for every $c \in C$. It has the derivation property and equals the differential on $\mathcal{A}$ resp. $\mathcal{B}$ upon the restriction on the corresponding sub- $C$-operad of $\mathcal{A} * \mathcal{B}$. Explicitly, for $T\left(x_{1}, \ldots, x_{n}\right) \in \mathcal{A} * \mathcal{B}$ with $x_{i} \in \overline{\mathcal{A}}$ or $\overline{\mathcal{B}}$, assuming (2), we have

$$
\partial\left(T\left(x_{1}, \ldots, x_{n}\right)\right)=\sum_{i=1}^{n}(-1)^{\sum_{j=1}^{i-1}\left|x_{j}\right|} T\left(x_{1}, \ldots, \partial\left(x_{i}\right), \ldots, x_{n}\right) .
$$

It is easily seen that the $\operatorname{dg}-C$-operad $(\mathcal{A} * \mathcal{B}, \partial)$ just described has the required universal property of the coproduct.

Now we are prepared to prove a version of (4) in a certain special case:

Lemma 2.4 Let $\left(\mathcal{A}, \partial_{\mathcal{A}}\right) \stackrel{\alpha}{\rightarrow}\left(\mathcal{A}^{\prime}, \partial_{\mathcal{A}^{\prime}}\right)$ and $\left(\mathcal{B}, \partial_{\mathcal{B}}\right) \stackrel{\beta}{\rightarrow}\left(\mathcal{B}^{\prime}, \partial_{\mathcal{B}^{\prime}}\right)$ be quisms of dg-C-operads, that is we assume homology of $\mathcal{A}, \mathcal{A}^{\prime}, \mathcal{B}, \mathcal{B}^{\prime}$ are graded $C$-operads and $H_{*}(\alpha), H_{*}(\beta)$ are graded $C$-operad isomorphisms. Then there are graded $C$-operad isomorphisms $\iota, \iota$ ' such that the following diagram commutes:

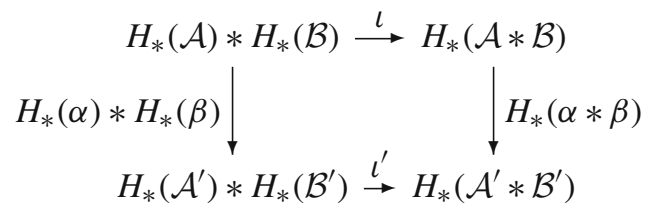

Proof Choose $\overline{\mathcal{A}}, \overline{\mathcal{B}}$ so that (5) and (6) hold. Now we want to choose $\overline{\mathcal{A}}^{\prime} \subset \mathcal{A}^{\prime}$ so that

$$
\begin{gathered}
\overline{\mathcal{A}}^{\prime} \oplus 1_{\mathcal{A}^{\prime}}=\mathcal{A}^{\prime}, \\
\alpha(\overline{\mathcal{A}}) \subset \overline{\mathcal{A}}^{\prime}
\end{gathered}
$$

and choose $\overline{\mathcal{B}}^{\prime} \subset \mathcal{B}^{\prime}$ similarly. To see that this is possible, we observe $\alpha(\overline{\mathcal{A}}) \cap 1_{\mathcal{A}^{\prime}}=0$ : If $\alpha(\bar{a}) \in 1_{\mathcal{A}^{\prime}}$ for some $\bar{a} \in \overline{\mathcal{A}}$, there is $u \in 1_{\mathcal{A}}$ such that $\alpha(u)=\alpha(\bar{a})$, hence $\alpha(\bar{a}-u)=0$ and $\partial_{\mathcal{A}}(\bar{a}-u)=0$ since both $\bar{a}$ and $u$ are of degree 0 . Since $\alpha$ is a 
quism, $\bar{a}-u=\partial_{\mathcal{A}} a$ for some $a \in \mathcal{A}$ and by the property (6) of $\overline{\mathcal{A}}$ we have $\bar{a}-u \in \overline{\mathcal{A}}$. But this implies $u \in \overline{\mathcal{A}}$, a contradiction.

Now use the explicit description (8) of the free product $\mathcal{A} * \mathcal{B}$ and the usual Künneth formula (3) to obtain an isomorphism

$$
H_{*}(\mathcal{A}) * H_{*}(\mathcal{B})=\bigoplus_{T} \bigotimes_{v} H_{*}(\overline{\mathcal{P}}(v)) \stackrel{\iota}{\rightarrow} H_{*}\left(\bigoplus_{T} \bigotimes_{v} \overline{\mathcal{P}}(v)\right)=H_{*}(\mathcal{A} * \mathcal{B})
$$

and similarly for $\mathcal{A}^{\prime}, \mathcal{B}^{\prime}$.

Assume we are given a free product tree $T$ and its vertex $v$. The tree $T$ comes equipped with $\overline{\mathcal{P}}$ as in (7). Let

$$
\overline{\mathcal{P}}^{\prime}(v):=(\overline{\mathcal{P}}(v))^{\prime}= \begin{cases}\overline{\mathcal{A}}^{\prime} & \text { for } \overline{\mathcal{P}}(v)=\overline{\mathcal{A}} \\ \overline{\mathcal{B}}^{\prime} & \text { for } \overline{\mathcal{P}}(v)=\overline{\mathcal{B}}\end{cases}
$$

and define a map

$$
\begin{aligned}
& \pi(v): \overline{\mathcal{P}}(v) \rightarrow \overline{\mathcal{P}}^{\prime}(v), \\
& \pi(v)= \begin{cases}\alpha & \text { for } \overline{\mathcal{P}}(v)=\overline{\mathcal{A}} \\
\beta & \text { for } \overline{\mathcal{P}}(v)=\overline{\mathcal{B}}\end{cases}
\end{aligned}
$$

This is justified by (9). Then the following diagram

$$
\begin{gathered}
\bigoplus_{T} \bigotimes_{v} H_{*}(\overline{\mathcal{P}}(v)) \stackrel{\iota}{\rightarrow} H_{*}\left(\bigoplus_{T} \bigotimes_{v} \overline{\mathcal{P}}(v)\right) \\
\bigoplus_{T} \otimes_{v} H_{*}(\pi(v)) \downarrow \\
\bigoplus_{*}\left(\bigoplus_{T} \bigotimes_{v} \pi(v)\right) \\
\bigoplus_{T} \bigotimes_{v} H_{*}\left(\overline{\mathcal{P}}^{\prime}(v)\right) \stackrel{\iota^{\prime}}{\rightarrow} H_{*}\left(\bigoplus_{T} \bigotimes_{v} \overline{\mathcal{P}}^{\prime}(v)\right)
\end{gathered}
$$

commutes by the naturality of the usual Künneth formula. The horizontal $C$-collection isomorphism $\iota$ is given in terms of tree compositions by the formula

$$
\iota\left(T\left(\left[x_{1}\right],\left[x_{2}\right], \ldots\right)\right)=\left[T\left(x_{1}, x_{2}, \ldots\right)\right]
$$

where $x_{1}, x_{2}, \ldots \in \overline{\mathcal{A}}$ or $\overline{\mathcal{B}}$. Now we verify that $\iota$ preserves the operadic composition:

$$
\iota\left(T_{x}\left(\left[x_{1}\right], \ldots\right)\right) \circ_{i} \iota\left(T_{y}\left(\left[y_{1}\right], \ldots\right)\right)=\iota\left(T_{x}\left(\left[x_{1}\right], \ldots\right) \circ_{i} T_{y}\left(\left[y_{1}\right], \ldots\right)\right)
$$

The left-hand side equals $\left[T_{x}\left(x_{1}, \ldots\right) \circ_{i} T_{y}\left(y_{1}, \ldots\right)\right]$, so we check

$$
\left[T_{x}\left(x_{1}, \ldots\right) \circ_{i} T_{y}\left(y_{1}, \ldots\right)\right]=\iota\left(T_{x}\left(\left[x_{1}\right], \ldots\right) \circ_{i} T_{y}\left(\left[y_{1}\right], \ldots\right)\right) .
$$


We would like to perform the same reducing operations on $T_{x}\left(x_{1}, \ldots\right) \circ_{i} T_{y}\left(y_{1}, \ldots\right)$ and $T_{x}\left(\left[x_{1}\right], \ldots\right) \circ_{i} T_{y}\left(\left[y_{1}\right], \ldots\right)$ parallely. For the first reducing operation, this is OK. For the second one, if, say, $\left[x_{1}\right] \in 1_{H_{*}(\mathcal{A})}$, then $x_{1}=u+\partial_{\mathcal{A}} a$ for some $u \in$ $1_{\mathcal{A}}$ and $a \in \mathcal{A}$. Hence $T_{x}\left(x_{1}, \ldots\right)=T_{x}(u, \ldots)+T_{x}\left(\partial_{\mathcal{A}} a, \ldots\right)$. So we can go on with $T_{x}(u, \ldots) \circ_{i} T_{y}\left(y_{1}, \ldots\right)$ and $T_{x}\left(\left[x_{1}\right], \ldots\right) \circ_{i} T_{y}\left(\left[y_{1}\right], \ldots\right)$, omitting the vertex $v_{1}$ decorated by $u$ resp. $\left[x_{1}\right]$, but we also have to apply the reducing operations to $T_{x}\left(\partial_{\mathcal{A}} a, \ldots\right) \circ_{i} T_{y}\left(y_{1}, \ldots\right)$. As it turns out, this tree composition is a boundary in $\mathcal{A} * \mathcal{B}$. We leave the details to the reader.

\section{Operadic cohomology of algebras}

\subsection{Reminder}

Let $\left(\mathcal{R}, \partial_{\mathcal{R}}\right) \stackrel{\rho}{\rightarrow}\left(\mathcal{A}, \partial_{\mathcal{A}}\right)$ be dg-C-operad over $\left(\mathcal{A}, \partial_{\mathcal{A}}\right)$, i.e. $\rho$ is a dg-C-operad morphism. Let $\left(\mathcal{M}, \partial_{\mathcal{M}}\right)$ be a $\operatorname{dg}-\mathcal{A}$-module. Define a $k$-module

$$
\operatorname{Der}_{\mathcal{A}}^{n}(\mathcal{R}, \mathcal{M})
$$

consisting of all $C$-collection morphisms $\theta: \mathcal{R} \rightarrow \mathcal{M}$ of degree $|\theta|=n$ in all colours satisfying

$$
\theta\left(r_{1} \circ_{i} r_{2}\right)=\theta\left(r_{1}\right) \circ_{i}^{R} \rho\left(r_{2}\right)+(-1)^{|\theta|\left|r_{1}\right|} \rho\left(r_{1}\right) \circ_{i}^{L} \theta\left(r_{2}\right)
$$

for any $r_{1}, r_{2} \in \mathcal{R}$ and any $1 \leqslant i \leqslant \operatorname{ar}\left(r_{1}\right)$. Denote

$$
\operatorname{Der}_{\mathcal{A}}(\mathcal{R}, \mathcal{M}):=\bigoplus_{n \in \mathbb{Z}} \operatorname{Der}_{\mathcal{A}}^{n}(\mathcal{R}, \mathcal{M})
$$

For $\theta \in \operatorname{Der}_{\mathcal{A}}(\mathcal{R}, \mathcal{M})$ homogeneous, let

$$
\delta \theta:=\theta \partial_{\mathcal{R}}-(-1)^{|\theta|} \partial_{\mathcal{M}} \theta
$$

Extending by linearity, the above formula defines a map $\delta$ with the $k$-module $\operatorname{Der}_{\mathcal{A}}$ $(\mathcal{R}, \mathcal{M})$ as a source.

Lemma 3.1 $\delta$ maps derivations to derivations and $\delta^{2}=0$.

Proof The degree of $\delta$ obviously equals -1 and

$$
\begin{aligned}
\delta^{2} \theta & =\left(\theta \partial_{\mathcal{R}}-(-1)^{|\theta|} \partial_{\mathcal{M}} \theta\right) \partial_{\mathcal{R}}-(-1)^{|\delta \theta|} \partial_{\mathcal{M}}\left(\theta \partial_{\mathcal{R}}-(-1)^{|\theta|} \partial_{\mathcal{M}} \theta\right) \\
& =\theta \partial_{\mathcal{R}}^{2}-(-1)^{|\theta|} \partial_{\mathcal{M}} \theta \partial_{\mathcal{R}}-(-1)^{|\theta|+1} \partial_{\mathcal{M}} \theta \partial_{\mathcal{R}}-(-1)^{|\theta|+1+|\theta|+1} \partial_{\mathcal{M}}^{2} \theta \\
& =0 .
\end{aligned}
$$


The following computation shows that $\delta$ maps derivations to derivations:

$$
\begin{aligned}
(\delta \theta)\left(r_{1} \circ_{i} r_{2}\right)= & \theta\left(\partial r_{1} \circ_{i} r_{2}+(-1)^{\left|r_{1}\right|} r_{1} \circ_{i} \partial r_{2}\right)+ \\
& -(-1)^{|\theta|} \partial\left(\theta r_{1} \circ_{i} \rho r_{2}+(-1)^{|\theta|\left|r_{1}\right|} \rho r_{1} \circ_{i} \theta r_{2}\right) \\
= & \theta \partial r_{1} \circ_{i} \rho r_{2}+(-1)^{|\theta|\left(\left|r_{1}\right|+1\right)} \rho \partial r_{1} \circ_{i} \theta r_{2} \\
& +(-1)^{\left|r_{1}\right|} \theta r_{1} \circ_{i} \rho \partial r_{2}+(-1)^{(|\theta|+1)\left|r_{1}\right|} \rho r_{1} \circ_{i} \theta \partial r_{2}+ \\
& -(-1)^{|\theta|} \partial \theta r_{1} \circ_{i} \rho r_{2}-(-1)^{\left|r_{1}\right|} \theta r_{1} \circ_{i} \partial \rho r_{2}+ \\
& -(-1)^{|\theta|\left(\left|r_{1}\right|+1\right)} \partial \rho r_{1} \circ_{i} \theta r_{2}-(-1)^{(|\theta|+1)\left|r_{1}\right|+|\theta|} \rho r_{1} \circ_{i} \partial \theta r_{2} \\
= & (\delta \theta) r_{1} \circ_{i} \rho r_{2}+(-1)^{|\delta \theta| \cdot\left|r_{1}\right|} \rho r_{1} \circ_{i}(\delta \theta) r_{2}
\end{aligned}
$$

where we have omitted the subscripts of $\partial$.

A particular example of this construction is

$$
\left(\mathcal{R}, \partial_{\mathcal{R}}\right) \underset{\rho}{\stackrel{\sim}{\rightarrow}}\left(\mathcal{A}, \partial_{\mathcal{A}}\right)
$$

a cofibrant [11] resolution of a $\mathrm{dg}-C$-operad $\mathcal{A}$, and

$$
\mathcal{M}:=\left(\mathcal{E}_{n d_{\mathcal{A}}}, \partial_{\mathcal{E} n d_{A}}\right)
$$

which is a dg- $\mathcal{A}$-module via a dg- $C$-operad morphism

$$
\left(\mathcal{A}, \partial_{\mathcal{A}}\right) \stackrel{\alpha}{\rightarrow}\left(\mathcal{E} n d_{A}, \partial_{\mathcal{E} n d_{A}}\right)
$$

determining an $\mathcal{A}$-algebra structure on a dg-k-module $\left(A, \partial_{A}\right)=\bigoplus_{c \in C}\left(A_{c}, \partial_{A_{c}}\right)$.

Let $\uparrow C$ denote the suspension of a graded object $C$, that is $(\uparrow C)_{n}:=C_{n-1}$. Analogously $\downarrow$ denotes the desuspension.

\section{Definition 3.2}

$$
\left(C^{*}(A, A), \delta\right):=\uparrow\left(\operatorname{Der}^{-*}\left(\left(\mathcal{R}, \partial_{\mathcal{R}}\right), \mathcal{E} n d_{A}\right), \delta\right)
$$

is called operadic cotangent complex of the $\mathcal{A}$-algebra $A$ and

$$
H^{*}(A, A):=H^{*}\left(C^{*}(A, A), \delta\right)
$$

is called operadic cohomology of $\mathcal{A}$-algebra $A$.

The change of grading $* \mapsto 1-*$ is purely conventional. For example, if $\mathcal{A}$ is the operad for associative algebras and $\mathcal{R}$ is its minimal resolution, under our convention we recover the grading of the Hochschild complex for which the bilinear cochains are of degree 1. 
3.2 Algebras with derivation

Let $\mathcal{A}$ be a dg-C-operad. Consider a $C$-collection $\Phi:=k\left\langle\phi_{c} \mid c \in C\right\rangle$, such that $\phi_{c}$ is of arity 1 , degree 0 and the input and output colours are both $c$. Let $\mathfrak{D}$ be the ideal in $\mathcal{A} * \mathbb{F}(\Phi)$ generated by all elements

$$
\phi_{c} \circ_{1} \alpha-\sum_{i=1}^{n} \alpha \circ_{i} \phi_{c_{i}}
$$

for $n \in \mathbb{N}_{0}, c, c_{1}, \ldots, c_{n} \in C$ and $\alpha \in \mathcal{A}\left(\begin{array}{c}c \\ c_{1}, \ldots, c_{n}\end{array}\right)$. Denote

$$
\mathcal{D} \mathcal{A}:=\left(\frac{\mathcal{A} * \mathbb{F}(\Phi)}{\mathfrak{D}}, \partial_{\mathcal{D} \mathcal{A}}\right)
$$

where $\partial_{\mathcal{D} \mathcal{A}}$ is the derivation given by the formulas

$$
\partial_{\mathcal{D A}}(a):=\partial_{\mathcal{A}}(a), \partial_{\mathcal{D} \mathcal{A}}\left(\phi_{c}\right):=0
$$

for $a \in \mathcal{A}$ and $c \in C$.

An algebra over $\mathcal{D} \mathcal{A}$ is a pair $(A, \phi)$, where $A=\bigoplus_{c \in C} A_{c}$ is an algebra over $\mathcal{A}$ and $\phi$ is a derivation of $A$ in the following sense: $\phi$ is a collection of degree $0 \mathrm{dg}$-maps $\phi_{c}: A_{c} \rightarrow A_{c}$ such that

$$
\phi_{c}\left(\alpha\left(a_{1}, \ldots, a_{n}\right)\right)=\sum_{i=1}^{n} \alpha\left(a_{1}, \ldots, \phi_{c_{i}}\left(a_{i}\right), \ldots, a_{n}\right)
$$

for $\alpha \in \mathcal{A}\left(\begin{array}{c}c \\ c_{1}, \ldots, c_{n}\end{array}\right)$ and $a_{j} \in A_{c_{j}}, 1 \leqslant j \leqslant n$.

Given a free resolution

$$
\mathcal{R}:=\left(\mathbb{F}(X), \partial_{\mathcal{R}}\right) \stackrel{\rho_{\mathcal{R}}}{\longrightarrow}\left(\mathcal{A}, \partial_{\mathcal{A}}\right),
$$

where $X$ is a $\mathrm{dg}$ - $C$-collection, it is surprisingly easy to explicitly construct a free resolution of $\left(\mathcal{D} \mathcal{A}, \partial_{\mathcal{D} \mathcal{A}}\right)$. Consider the free graded $C$-operad

$$
\mathcal{D} \mathcal{R}:=\mathbb{F}(X \oplus \Phi \oplus \underline{X}),
$$

where $\underline{X}:=\uparrow X$. We denote by $\underline{x}$ the element $\uparrow x \in \underline{X}$ corresponding to $x \in X$. To describe the differential, let $s: \mathbb{F}(X) \rightarrow \mathcal{D} \mathcal{R}$ be a degree +1 derivation determined by

$$
s(x):=\underline{x} \text { for } x \in X
$$


Then define a degree -1 derivation $\partial_{\mathcal{D} \mathcal{R}}: \mathcal{D} \mathcal{R} \rightarrow \mathcal{D} \mathcal{R}$ by

$$
\begin{aligned}
\partial_{\mathcal{D} \mathcal{R}}(x) & :=\partial_{\mathcal{R}}(x), \\
\partial_{\mathcal{D} \mathcal{R}}\left(\phi_{c}\right) & :=0, \\
\partial_{\mathcal{D} \mathcal{R}}(\underline{x}) & :=\phi_{c} \circ_{1} x-\sum_{i=0}^{n} x \circ_{i} \phi_{c_{i}}-s\left(\partial_{\mathcal{R}} x\right) .
\end{aligned}
$$

Convention 3.3 From now on we will assume

$$
n \in \mathbb{N}_{0}, c, c_{1}, \ldots, c_{n} \in C, x \in X\left(\begin{array}{c}
c \\
c_{1}, \ldots, c_{n}
\end{array}\right)
$$

whenever any of these symbols appears. We will usually omit the lower indices $c$ and $c_{i}$ 's for $\phi$.

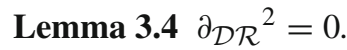

Proof Using the tree compositions of Sect. 2.2, let $\partial_{\mathcal{R}}(x)=\sum_{i} T_{i}\left(x_{i 1}, \ldots, x_{i n_{i}}\right)$.

$$
\begin{aligned}
\partial_{\mathcal{D R}}{ }^{2}(x)= & \partial_{\mathcal{D} \mathcal{R}}\left(\phi \circ_{1} x-\sum_{j=1}^{n} x \circ_{j} \phi-s\left(\partial_{\mathcal{R}}(x)\right)\right) \\
= & \phi \circ_{1} \partial_{\mathcal{D} \mathcal{R}}(x)-\sum_{j=1}^{n} \partial_{\mathcal{D} \mathcal{R}}(x) \circ_{j} \phi+ \\
& -\partial_{\mathcal{D} \mathcal{R}}\left(\sum_{i} \sum_{j=1}^{n_{i}} \epsilon_{i j} T_{i}\left(x_{i 1}, \ldots, \underline{x_{i j}}, \ldots, x_{i n_{i}}\right)\right)
\end{aligned}
$$

If we assume (2), then $\epsilon_{i j}=(-1)^{\sum_{l=1}^{j-1}\left|x_{i l}\right|}$. The last application of $\partial_{\mathcal{D} \mathcal{R}}$ on the double sum can be rewritten as

$$
\begin{aligned}
& \sum_{i} \sum_{j=1}^{n_{i}} \sum_{\substack{1 \leqslant k \leqslant n_{i} \\
k \neq j}} \tilde{\epsilon}_{i j k} T_{i}\left(x_{i 1}, \ldots, \partial_{\mathcal{R}}\left(x_{i k}\right), \ldots, x_{i j}, \ldots, x_{i n_{i}}\right) \\
& \quad+\sum_{i} \sum_{j=1}^{n_{i}} T_{i}\left(x_{i 1}, \ldots, \phi \circ_{1} x_{i j}, \ldots, x_{i n_{i}}\right)+ \\
& \quad-\sum_{i} \sum_{j=1}^{n_{i}} \sum_{k=1}^{\operatorname{ar}\left(x_{i j}\right)} T_{i}\left(x_{i 1}, \ldots, x_{i j} \circ_{k} \phi, \ldots, x_{i n_{i}}\right)+ \\
& \quad-\sum_{i} \sum_{j=1}^{n_{i}} T_{i}\left(x_{i 1}, \ldots, s\left(\partial_{\mathcal{R}}\left(x_{i j}\right)\right), \ldots, x_{i n_{i}}\right)
\end{aligned}
$$


where $\tilde{\epsilon}_{i j k}=\epsilon_{i j} \epsilon_{i k}$ if $k<j$ and $\tilde{\epsilon}_{i j k}=-\epsilon_{i j} \epsilon_{i k}$ if $k>j$. The second and third lines sum to

$$
\begin{aligned}
& \sum_{i} \phi \circ_{1} T_{i}\left(x_{i 1}, \ldots, x_{i n_{i}}\right)-\sum_{i} \sum_{j=1}^{\operatorname{ar}\left(T_{i}\right)} T_{i}\left(x_{i 1}, \ldots, x_{i n_{i}}\right) \circ_{j} \phi \\
& =\phi \circ_{1} \partial_{\mathcal{D} \mathcal{R}}(x)-\sum_{j=1}^{n} \partial_{\mathcal{D} \mathcal{R}}(x) \circ_{j} \phi,
\end{aligned}
$$

while the first and last rows sum to

$$
-s \partial_{\mathcal{D} \mathcal{R}}\left(\sum_{i} T_{i}\left(x_{i 1}, \ldots, x_{i n_{i}}\right)\right)=-s \partial_{\mathcal{D} \mathcal{R}}{ }^{2}=0
$$

and this concludes the computation.

From now on, we will refer by $\mathcal{D} \mathcal{R}$ also to the $d g-C$-operad $\left(\mathcal{D} \mathcal{R}, \partial_{\mathcal{D} \mathcal{R}}\right)$. Define a $C$-operad morphism $\rho_{\mathcal{D} \mathcal{R}}: \mathcal{D} \mathcal{R} \rightarrow \mathcal{D} \mathcal{A}$ by

$$
\begin{aligned}
\rho_{\mathcal{D} \mathcal{R}}(x) & :=\rho_{\mathcal{R}}(x), \\
\rho_{\mathcal{D} \mathcal{R}}\left(\phi_{c}\right) & :=\phi_{c}, \\
\rho_{\mathcal{D} \mathcal{R}}(\underline{x}) & :=0 .
\end{aligned}
$$

Theorem $3.5 \rho_{\mathcal{D} \mathcal{R}}$ is a free resolution of $\mathcal{D} \mathcal{A}$.

Example 3.6 Let's see what we get for $\mathcal{A}:=\mathcal{A} s s=\mathbb{F}(\mu) /\left(\mu \circ_{1} \mu-\mu \circ_{2} \mu\right)$ and its minimal resolution (see e.g. [12] $) \mathcal{R}:=\mathcal{A} s s_{\infty}=\left(\mathbb{F}(X), \partial_{\mathcal{R}}\right) \stackrel{\rho_{\mathcal{R}}}{\longrightarrow}(\mathcal{A s s}, 0)$, where

$$
X=k\left\langle x^{2}, x^{3}, \ldots\right\rangle
$$

is the collection spanned by $x^{n}$ in arity $n$ and degree $\left|x^{n}\right|=n-2$ and $\partial_{\mathcal{R}}$ is a derivation differential given by

$$
\partial_{\mathcal{R}}\left(x^{n}\right):=\sum_{i+j=n+1} \sum_{k=1}^{i}(-1)^{i+(k+1)(j+1)} x^{i} \circ_{k} x^{j}
$$

and the quism $\rho_{\mathcal{R}}: \mathcal{R}=\mathcal{A} s s_{\infty} \rightarrow \mathcal{A} s s=\mathcal{A}$ is given by

$$
\rho_{\mathcal{R}}\left(x^{2}\right):=\mu, \rho_{\mathcal{R}}\left(x^{n}\right):=0 \text { for } n \geqslant 3
$$

Then the associated operad with derivation is

$$
\mathcal{D} \mathcal{A}:=\frac{\mathcal{A s s} * \mathbb{F}(\Phi)}{\left(\phi \circ \mu-\mu \circ_{1} \phi-\mu \circ_{2} \phi\right)},
$$


where $\Phi:=k\langle\phi\rangle$ with $\phi$ a generator of arity 1 . Its free resolution is

$$
\mathcal{D} \mathcal{R}:=\left(\mathbb{F}(X \oplus \Phi \oplus \underline{X}), \partial_{\mathcal{D} \mathcal{R}}\right) \stackrel{\rho_{\mathcal{D} \mathcal{R}}}{\longrightarrow}(\mathcal{D} \mathcal{A}, 0),
$$

where the differential $\partial_{\mathcal{D} \mathcal{R}}$ is given by

$$
\begin{aligned}
& \partial_{\mathcal{D} \mathcal{R}}(x):=\partial_{\mathcal{R}}(x), \\
& \partial_{\mathcal{D} \mathcal{R}}(\phi):=0, \\
& \partial_{\mathcal{D} \mathcal{R}}\left(\underline{x}^{n}\right):=\phi \circ_{1} x^{n}-\sum_{i=1}^{n} x^{n} \circ_{i} \phi-\sum_{i+j=n+1} \sum_{k=1}^{i}(-1)^{i+(k+1)(j+1)} \\
& \left(\underline{x}^{i} \circ_{k} x^{j}+(-1)^{i} x^{i} \circ_{k} \underline{x}^{j}\right)
\end{aligned}
$$

and the quism $\rho_{\mathcal{D} \mathcal{R}}$ by

$$
\rho_{\mathcal{D} \mathcal{R}}(x):=\rho_{\mathcal{R}}(x), \rho_{\mathcal{D} \mathcal{R}}(\phi):=\phi, \rho_{\mathcal{D} \mathcal{R}}(\underline{x})=0 .
$$

Proof of Theorem 3.5 Obviously $\rho_{\mathcal{D} \mathcal{R}}$ has degree 0 and commutes with differentials because of the relations in $\mathcal{D} \mathcal{A}$. Let's abbreviate $\partial_{\mathcal{D} \mathcal{R}}=: \partial$. First we want to use a spectral sequence to split $\partial$ such that $\partial^{0}$, the 0 th page part of $\partial$, is nontrivial only on the generators from $\underline{X}$.

Let's put an additional grading gr on the $C$-collection $X \oplus \Phi \oplus \underline{X}$ of generators:

$$
\operatorname{gr}(x):=|x|, \operatorname{gr}(\phi):=1, \operatorname{gr}(\underline{x}):=|\underline{x}| .
$$

This induces a grading on $\mathcal{D} \mathcal{R}$ determined by the requirement that the composition is of gr degree 0 . Let

$$
\mathfrak{F}_{p}:=\bigoplus_{i=0}^{p}\{z \in \mathcal{D} \mathcal{R} \mid \operatorname{gr}(z)=i\}
$$

Obviously $\partial_{\mathcal{D} \mathcal{R}} \mathfrak{F}_{p} \subset \mathfrak{F}_{p}$. Consider the spectral sequence $E^{*}$ associated to the filtration

$$
0 \hookrightarrow \mathfrak{F}_{0} \hookrightarrow \mathfrak{F}_{1} \hookrightarrow \cdots
$$

of $\mathcal{D} \mathcal{R}$. On $\mathcal{D} \mathcal{A}$ we have the trivial filtration

$$
0 \hookrightarrow \mathcal{D} \mathcal{A}
$$

and the associated spectral sequence $E^{\prime *}$.

We will show that $\rho_{\mathcal{D} \mathcal{R}}$ induces quism $\left(E^{1}, \partial^{1}\right) \stackrel{\sim}{\rightarrow}\left(E^{\prime 1}, \partial^{\prime 1}\right)$. Then we can use the comparison theorem since both filtrations are obviously bounded below and exhaustive (e.g. [20, p. 126, Theorem 5.2.12, and p. 135, Theorem 5.5.1]). 
Then the 0th page satisfies $E^{0} \cong \mathbb{F}(X \oplus \Phi \oplus \underline{X})$ and is equipped with the derivation differential $\partial^{0}$ :

$$
\partial^{0}(x)=0=\partial^{0}\left(\phi_{c}\right), \partial^{0}(\underline{x})=\phi_{c} \circ_{1} x-\sum_{i=1}^{\operatorname{ar}(x)} x \circ_{i} \phi_{c_{i}} .
$$

Denote by $\mathfrak{D}$ the ideal in $\mathbb{F}(X \oplus \Phi)$ generated by

$$
\phi_{c} \circ_{1} x-\sum_{i=1}^{\operatorname{ar}(x)} x \circ_{i} \phi_{c_{i}}
$$

for all $x \in X\left(\begin{array}{c}c \\ c_{1}, \ldots, c_{\operatorname{ar}(x)}\end{array}\right)$ of arbitrary colours.

\section{Sublemma 3.7}

$$
H_{*}\left(E^{0}, \partial^{0}\right) \cong \frac{\mathbb{F}(X \oplus \Phi)}{\mathfrak{D}}
$$

Once this sublemma is proved, $\partial^{1}$ on $E^{1} \cong H_{*}\left(E^{0}, \partial^{0}\right) \cong \frac{\mathbb{F}(X \oplus \Phi)}{\mathfrak{D}}$ will be given by

$$
\partial^{1}(x)=\partial_{\mathcal{R}}(x), \quad \partial^{1}\left(\phi_{c}\right)=0 .
$$

We immediately see that $E^{\prime 1} \cong \mathcal{D} \mathcal{A}$ and it is equipped with the differential $\partial^{\prime 1}=\partial_{\mathcal{D} \mathcal{A}}$. To see that $\rho_{\mathcal{D} \mathcal{R}}{ }^{1}: E^{1} \rightarrow E^{\prime 1}$ induced by $\rho_{\mathcal{D} \mathcal{R}}$ is a quism, observe that we can use the relations (12) in $\mathcal{D} \mathcal{A}$ to "move all the $\phi$ 's to the bottom of the tree compositions", hence, denoting

$$
\Phi^{\prime}:=\mathbb{F}(\Phi)
$$

we have

$$
\mathcal{D} \mathcal{A} \cong \mathcal{A} \circ \Phi^{\prime}
$$

The composition and the differential on $\mathcal{A} \circ \Phi^{\prime}$ are transferred along this isomorphism from $\mathcal{D} \mathcal{A}$. Similarly,

$$
\frac{\mathbb{F}(X \oplus \Phi)}{\mathfrak{D}} \cong \mathbb{F}(X) \circ \Phi^{\prime}
$$

Under these quisms

$$
\rho_{\mathcal{D} \mathcal{R}}{ }^{1} \text { becomes } \rho_{\mathcal{R}} \circ 1_{\Phi^{\prime}}
$$

It remains to use the usual Künneth formula (3) to finish the proof of Theorem 3.5. 
Proof of Sublemma 3.7 Denote $\phi_{c}^{m}:=\phi_{c} \circ_{1} \cdots \circ_{1} \phi_{c}$ the $m$-fold composition of $\phi_{c}$. Let

$$
\mathcal{D} \mathcal{R}^{0}:=\mathbb{F}(X \oplus \Phi)
$$

and, for $n \geqslant 0$, let $\mathcal{D} \mathcal{R}^{n+1} \subset \mathcal{D} \mathcal{R}$ be spanned by elements

$$
\begin{aligned}
& \phi_{c}^{m} \circ_{1} x \circ\left(x_{1}, \ldots, x_{\operatorname{ar}(x)}\right) \text { and } \\
& \phi_{c}^{m} \circ_{1} \underline{x} \circ\left(x_{1}, \ldots, x_{\operatorname{ar}(x)}\right)
\end{aligned}
$$

for all $x \in X\left(\begin{array}{c}c \\ c_{1}, \ldots, c_{\operatorname{ar}(x)}\end{array}\right), m \geqslant 0, x_{i} \in \mathcal{D R}^{n}\left(\begin{array}{c}c_{i} \\ \ldots\end{array}\right), 1 \leqslant i \leqslant \operatorname{ar}(x)$. In other words,

$$
\mathcal{D} \mathcal{R}^{n+1}=\Phi^{\prime} \circ(X \oplus \underline{X}) \circ \mathcal{D} \mathcal{R}^{n} .
$$

$\mathcal{D} \mathcal{R}^{n}$ is obviously closed under $\partial^{0}$ and

$$
\mathcal{D R}^{0} \hookrightarrow \mathcal{D R}^{1} \hookrightarrow \cdots \rightarrow \operatorname{colim}_{n} \mathcal{D} \mathcal{R}^{n} \cong \mathcal{D} \mathcal{R},
$$

where the colimit is taken in the category of $\mathrm{dg}$ - $C$-collections.

Before we go further, we must make a short notational digression. Let $T\left(g_{1}, \ldots, g_{m}\right)$ be a tree composition with $g_{i} \in X \sqcup \Phi \sqcup \underline{X}$ for $1 \leqslant i \leqslant m$. Recall the tree $T$ has vertices $v_{1}, \ldots, v_{m}$ decorated by $g_{1}, \ldots, g_{m}$ (in that order). We say that $g_{j}$ is in depth $d$ in $T\left(g_{1}, \ldots, g_{j}, \ldots, g_{m}\right)$ iff the shortest path from $v_{j}$ to the root vertex passes through exactly $d$ vertices (including $v_{j}$ and the root vertex) decorated by elements of $X \sqcup \underline{X}$.

As an example, consider

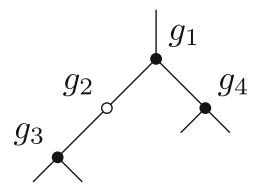

If $g_{1}, g_{3}, g_{4} \in X$ and $g_{2} \in \Phi$, then $g_{1}, g_{2}$ are in depth 1 and $g_{3}, g_{4}$ are in depth 2 .

Using the notion of depth, the definition of $\mathcal{D} \mathcal{R}^{n}$ can be rephrased as follows: $\mathcal{D} \mathcal{R}^{n}$ is spanned by $T\left(g_{1}, \ldots, g_{m}\right)$ with $g_{i} \in X \sqcup \Phi \sqcup \underline{X}, 1 \leqslant i \leqslant m$, such that if $g_{j} \in \underline{X}$ for some $j$, then $g_{j}$ is in depth $\leqslant n$ in $T\left(g_{1}, \ldots, g_{m}\right)$.

Consider the quotient $\mathcal{Q}^{n}$ of $\mathbb{F}(X \oplus \Phi)$ by the ideal generated by elements

$$
T\left(g_{1}, \ldots, g_{j-1}, \phi_{c} \circ_{1} x_{j}-\sum_{i=0}^{\operatorname{ar}\left(x_{j}\right)} x_{j} \circ_{i} \phi_{c_{i}}, g_{j+1}, \ldots, g_{m}\right)
$$

for any tree $T$, any $g_{1}, \ldots, g_{j-1}, g_{j+1}, \ldots, g_{m} \in X \sqcup \Phi$ and any $x_{j} \in X$ in depth $\leqslant n$ in $T\left(g_{1}, \ldots, g_{j-1}, x_{j}, g_{j+1}, \ldots, g_{m}\right)$. There are obvious projections

$$
\mathbb{F}(X \oplus \Phi)=\mathcal{Q}^{0} \rightarrow \mathcal{Q}^{1} \rightarrow \cdots \rightarrow \operatorname{colim}_{n} \mathcal{Q}^{n} \cong \frac{\mathbb{F}(X \oplus \Phi)}{\mathfrak{D}} .
$$


To see the last isomorphism, observe that we can use the relations defining $\mathcal{Q}^{n}$ to "move" the $\phi_{c}$ 's in tree compositions so that they are all in depth $\geqslant n$ or in positions such that their inputs are leaves, then use (17).

For example, consider the following computation in $\mathcal{Q}^{2}$, where the black vertices are decorated by $X$ and white vertices by $\Phi$ :

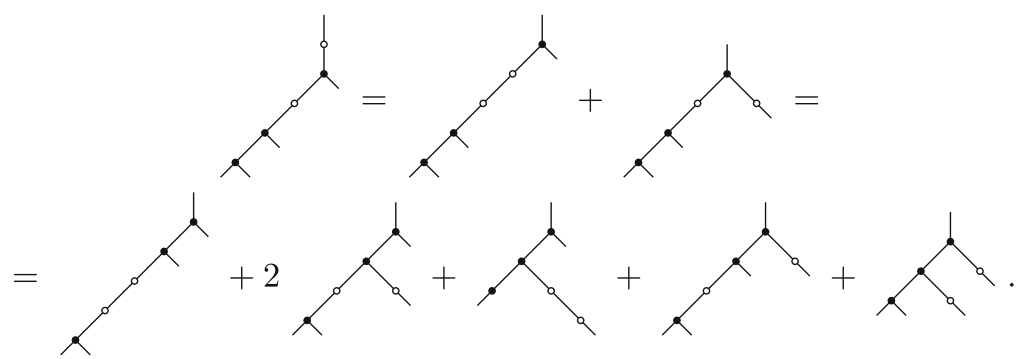

Notice that we can't get the white vertices any deeper in $\mathcal{Q}^{2}$.

In particular,

$$
\mathcal{Q}^{n+1} \cong X \circ \mathcal{Q}^{n}
$$

Obviously

$$
H_{*}\left(\mathcal{D} \mathcal{R}^{0}, \partial^{0}\right) \cong \mathcal{Q}^{0}
$$

and we claim that

$$
H_{*}\left(\mathcal{D} \mathcal{R}^{n}, \partial^{0}\right) \cong \mathcal{Q}^{n}
$$

for $n \geqslant 1$. Suppose the claim holds for $n$ and we prove it for $n+1$. The idea is to use a spectral sequence to get rid of the last sum in the formula

$$
\begin{aligned}
& \partial^{0}\left(\phi_{c}^{m} \circ_{1} \underline{x} \circ\left(x_{1}, \ldots, x_{\operatorname{ar}(x)}\right)\right)= \\
& =\phi_{c}^{m+1} \circ_{1} x \circ\left(x_{1}, \ldots, x_{\operatorname{ar}(x)}\right)+ \\
& \quad-\sum_{i=1}^{\operatorname{ar}(x)} \phi^{m} \circ_{1} x \circ_{i} \phi \circ\left(x_{1}, \ldots, x_{\operatorname{ar}(x)}\right)+ \\
& \quad+(-1)^{|\underline{x}|} \sum_{i=1}^{\operatorname{ar}(x)}(-1)^{\sum_{j=1}^{i-1}\left|x_{j}\right|} \phi^{m} \circ_{1} \underline{x} \circ\left(x_{1}, \ldots, \partial^{0}\left(x_{i}\right), \ldots, x_{\operatorname{ar}(x)}\right) .
\end{aligned}
$$

Consider the spectral sequence $E^{0 *}$ on $\mathcal{D} \mathcal{R}^{n+1}$ associated to the filtration

$$
0 \hookrightarrow \mathfrak{G}_{0} \hookrightarrow \mathfrak{G}_{1} \hookrightarrow \cdots \hookrightarrow \mathcal{D} \mathcal{R}^{n}
$$


where $\mathfrak{G}_{k}$ is spanned by

$$
\phi^{m} \circ_{1} g \circ\left(x_{1}, \ldots, x_{\operatorname{ar}(x)}\right)
$$

for all $m \geqslant 0, g \in X \oplus \underline{X}, x_{i} \in \mathcal{D} \mathcal{R}^{n}$ and $\sum_{i=1}^{\operatorname{ar}(x)}\left|x_{i}\right| \leqslant k$. Obviously $\partial^{0}: \mathfrak{G}_{k} \rightarrow \mathfrak{G}_{k}$.

We will use the comparison theorem for the obvious projection

$$
\mathcal{D} \mathcal{R}^{n+1} \stackrel{\mathrm{pr}}{\rightarrow} \mathcal{Q}^{n+1}
$$

We consider the zero differential on $\mathcal{Q}^{n+1}$. It is easily seen that $\operatorname{pr} \partial^{0}=0$, hence pr is dg-C-collection morphism. We equip $\mathcal{Q}^{n+1}$ with the trivial filtration $0 \hookrightarrow \mathcal{Q}^{n+1}$ and consider the associated spectral sequence $E^{\prime 0 *}$. Again, both filtrations are bounded below and exhaustive.

On the 0th page $E^{00} \cong \mathcal{D} \mathcal{R}^{n+1}$, the differential $\partial^{00}$ has the desired form:

$$
\begin{aligned}
\partial^{00}\left(\phi^{m} \circ_{1} \underline{x} \circ\left(x_{1}, \ldots, x_{\operatorname{ar}(x)}\right)\right)=\phi_{c}^{m+1} \circ_{1} x \circ\left(x_{1}, \ldots, x_{\operatorname{ar}(x)}\right)+ \\
\quad-\sum_{i=1}^{\operatorname{ar}(x)} \phi^{m} \circ_{1} x \circ_{i} \phi \circ\left(x_{1}, \ldots, x_{\operatorname{ar}(x)}\right)
\end{aligned}
$$

and $\partial^{00}$ is zero on other elements. For this differential $\partial^{00}$, it is (at last!) clear how its kernel looks (compare to $\left.\partial^{0}\right)$, namely Ker $\partial^{00}=\mathbb{F}(X \oplus \Phi) \circ \mathcal{D} \mathcal{R}^{n}$. Hence

$$
H_{*}\left(E^{00}, \partial^{00}\right) \cong X \circ \mathcal{D} \mathcal{R}^{n}
$$

This is $E^{01}$ and the differential $\partial^{01}$ is equal to the restriction of $\partial^{0}$ onto $X \circ \mathcal{D} \mathcal{R}^{n}$.

For $E^{\prime 0 *}$ everything is trivial, $E^{\prime 01} \cong \mathcal{Q}^{n+1}$ and $\partial^{\prime 01}=0$.

Then $\mathrm{pr}^{1}: E^{01} \rightarrow E^{\prime 01}$ induced by pr is quism, because

$$
H_{*}\left(E^{01}, \partial^{01}\right) \cong X \circ H_{*}\left(\mathcal{D} \mathcal{R}^{n}, \partial^{0}\right) \cong X \circ \mathcal{Q}^{n} \cong \mathcal{Q}^{n+1},
$$

where the first isomorphism follows from the usual Künneth formula (3), the second one follows from the induction hypothesis and the last one was already observed in (19).

This concludes the proof of the claim $H_{*}\left(\mathcal{D} \mathcal{R}^{n}, \partial^{0}\right) \cong \mathcal{Q}^{n}$. Finally

$$
H_{*}\left(\mathcal{D} \mathcal{R}, \partial^{0}\right) \cong H_{*}\left(\operatorname{colim}_{n} \mathcal{D} \mathcal{R}^{n}\right) \cong \operatorname{colim}_{n} H_{*}\left(\mathcal{D} \mathcal{R}^{n}\right) \cong \operatorname{colim}_{n} \mathcal{Q}^{n} \cong \frac{\mathbb{F}(X \oplus \Phi)}{\mathfrak{D}}
$$

proves Sublemma 3.7.

Now that the sublemma is proved, we easily go through all the isomorphisms to check (16) and (18). 


\subsection{Augmented cotangent complex}

Let

$$
\left(\mathcal{A}, \partial_{\mathcal{A}}\right) \stackrel{\alpha}{\rightarrow}\left(\mathcal{E} n d_{A}, \partial_{\mathcal{E}_{n d}}\right)
$$

be an $\mathcal{A}$-algebra structure on $A$. We begin by extracting the operadic cohomology from $\mathcal{D} \mathcal{R}$. Let $\mathbb{F}(X \oplus \Phi \oplus \underline{X}) \rightarrow \mathcal{A}$ be the dg-C-operad morphism which equals $\rho_{\mathcal{R}}$ on $X$ and vanishes on the other generators. Hence $\mathcal{D} \mathcal{R}=\mathbb{F}(X \oplus \Phi \oplus \underline{X})$ is a dg-C-operad over $\mathcal{A}$.

For $M$ one of the subsets $X, X \oplus \Phi, X \oplus \underline{X}$ of $\mathcal{D} \mathcal{R}$ define

$$
\operatorname{Der}_{\mathcal{A}}^{M}\left(\mathcal{D} \mathcal{R}, \mathcal{E} n d_{A}\right):=\left\{\theta \in \operatorname{Der}_{\mathcal{A}}\left(\mathcal{D} \mathcal{R}, \mathcal{E} n d_{A}\right) \mid \forall m \in M \quad \theta(m)=0\right\} .
$$

We will abbreviate this by $\operatorname{Der}^{M}$. Let $\bar{\delta}$ be the differential on $\operatorname{Der}^{M}$ defined by

$$
\bar{\delta} \theta:=\theta \partial_{\mathcal{D} \mathcal{R}}-(-1)^{|\theta|} \partial_{\mathcal{E} n d_{A}} \theta .
$$

A check similar to that for (10) verifies this is well defined. Obviously

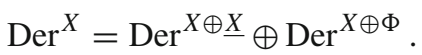

Recall we assume the $\operatorname{dg}-k$-module $A$ is graded by the colours, that is $A=\bigoplus_{c \in C} A_{c}$. Hence we have

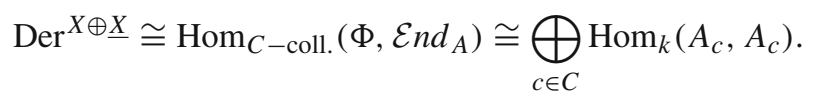

Importantly, $\operatorname{Der}^{X \oplus \Phi}$ is closed under $\bar{\delta}$.

\section{Lemma 3.8}

$$
\left(\operatorname{Der}^{X \oplus \Phi}, \bar{\delta}\right) \cong \downarrow\left(\operatorname{Der}_{\mathcal{A}}\left(\mathcal{R}, \mathcal{E} n d_{A}\right), \delta\right)
$$

as dg-k-modules.

Proof Recall $\downarrow \delta=-\delta$. Define a degree +1 map

$$
\operatorname{Der}^{X \oplus \Phi} \stackrel{f_{1}}{\rightarrow} \operatorname{Der}_{\mathcal{A}}\left(\mathcal{R}, \mathcal{E} n d_{A}\right)
$$

by the formula

$$
\left(f_{1} \theta^{\prime}\right)(x):=\theta^{\prime}(\underline{x}) \quad \text { for } \theta^{\prime} \in \operatorname{Der}^{X \oplus \Phi} .
$$

Its inverse, $f_{2}$ of degree -1 , is defined for $\theta \in \operatorname{Der}_{\mathcal{A}}\left(\mathcal{R}, \mathcal{E} n d_{A}\right)$ by the formulas

$$
\left(f_{2} \theta\right)(a)=0=\left(f_{2} \theta\right)(\phi), \quad\left(f_{2} \theta\right)(\underline{x})=\theta(x) .
$$


Obviously $f_{2} f_{1}=1$ and $f_{1} f_{2}=1$ and it remains to check $f_{1} \bar{\delta}=-\delta f_{1}$.

$$
\begin{aligned}
& \left(f_{1}\left(\bar{\delta} \theta^{\prime}\right)\right)(x)=\left(\bar{\delta} \theta^{\prime}\right)(\underline{x})=\theta^{\prime}\left(\partial_{\mathcal{D} \mathcal{R}} \underline{x}\right)-(-1)^{\left|\theta^{\prime}\right|} \partial_{\mathcal{E} n d_{A}}\left(\theta^{\prime}(\underline{x})\right), \\
& \left(-\delta\left(f_{1} \theta^{\prime}\right)\right)(x)=-\left(f_{1} \theta^{\prime}\right)\left(\partial_{\mathcal{R}} x\right)+(-1)^{\left|f_{1} \theta^{\prime}\right|} \partial_{\mathcal{E}_{n d_{A}}}\left(\left(f_{1} \theta^{\prime}\right)(x)\right) \text {. }
\end{aligned}
$$

Now we check $\theta^{\prime}\left(\partial_{\mathcal{D} \mathcal{R}} \underline{x}\right)=-\left(f_{1} \theta^{\prime}\right)\left(\partial_{\mathcal{R}} x\right)$. Let $\partial_{\mathcal{R}} x=\sum_{i} T_{i}\left(x_{i 1}, \ldots, x_{i n_{i}}\right)$.

$$
\begin{aligned}
& \theta^{\prime}\left(\partial_{\mathcal{D} \mathcal{R} \underline{x})=} \theta^{\prime}\left(\phi \circ x-\sum_{j} x \circ_{j} \phi-s\left(\partial_{\mathcal{R}} x\right)\right)\right. \\
&=-\theta^{\prime}\left(s \sum_{i} T_{i}\left(x_{i 1}, \ldots, x_{i n_{i}}\right)\right) \\
&=-\theta^{\prime}\left(\sum_{i} \sum_{j=1}^{n_{i}} \epsilon_{i j} T_{i}\left(x_{i 1}, \ldots, \underline{x_{i j}}, \ldots, x_{i n_{i}}\right)\right) \\
&=-\sum_{i} \sum_{j} \epsilon_{i j}^{1+\left|\theta^{\prime}\right|} T_{i}\left(\rho_{\mathcal{R}}\left(x_{i 1}\right), \ldots, \theta^{\prime} \underline{\left(x_{i j}\right)}, \ldots, \rho_{\mathcal{R}}\left(x_{i n_{i}}\right)\right), \\
&-\left(f_{1} \theta^{\prime}\right)\left(\partial_{\mathcal{R}} x\right)=\ldots=-\sum_{i} \sum_{j} \underline{\epsilon_{i j}^{\left|f_{1} \theta^{\prime}\right|}} T_{i}\left(\rho_{\mathcal{R}}\left(x_{i 1}\right), \ldots,\right. \\
&\left.\left(f_{1} \theta^{\prime}\right)\left(\underline{x_{i j}}\right), \ldots, \rho_{\mathcal{R}}\left(x_{i n_{i}}\right)\right),
\end{aligned}
$$

where we have denoted $\epsilon_{i j}:=(-1)^{\sum_{l=1}^{j-1}\left|x_{i l}\right|}$.

Definition 3.9 We call

$$
C_{\text {aug }}^{*}(A, A):=\left(\left(\operatorname{Der}^{X}\right)^{-*}, \bar{\delta}\right)
$$

augmented operadic cotangent complex of $A$ and its cohomology

$$
H_{\text {aug }}^{*}(A, A):=H^{*}\left(C_{\text {aug }}^{*}(A, A), \bar{\delta}\right)
$$

\section{augmented operadic cohomology of $A$.}

The interpretation of the augmentation $\left(\operatorname{Der}^{X \oplus \underline{X}}\right)^{-*} \stackrel{\bar{\delta}}{\rightarrow}\left(\operatorname{Der}^{X \oplus \Phi}\right)^{-*} \cong C^{*}(A, A)$ of the usual cotangent complex $C^{*}(A, A)$ is via infinitesimal automorphisms of the $\mathcal{A}$-algebra structure on $A$. This suggests a relation between $H_{\text {aug }}^{*}(A, A)$ and $H^{*}(A, A)$. It is best seen in an example:

Example 3.10 Continuing Example 3.6, let $A$ be $k$-module with a structure of an associative algebra, that is

$$
\mathcal{A} s s \stackrel{\alpha}{\rightarrow} \mathcal{E}_{n d} \text {. }
$$


We have

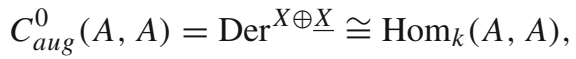

$$
\begin{aligned}
& C_{\text {aug }}^{n}(A, A)=\left(\operatorname{Der}^{X \oplus \Phi}\right)^{-n} \cong \operatorname{Hom}_{C-\operatorname{coll}}\left(\underline{X}, \mathcal{E}_{n d_{A}}\right)^{-n} \\
& \cong \operatorname{Hom}_{C-\text { coll. }}\left(\underline{X}_{n}, \mathcal{E} n d_{A}\right) \cong \mathcal{E}_{n d_{A}}(n+1)=\operatorname{Hom}_{k}\left(A^{\otimes n+1}, A\right)
\end{aligned}
$$

and, for $f \in C_{\text {aug }}^{n}(A, A)$,

$$
\bar{\delta} f=(-1)^{n+1} \mu \circ_{2} f+\sum_{k=1}^{n}(-1)^{n+1-k} f \circ_{k} \mu+\mu \circ_{1} f .
$$

So the augmented cotangent complex is the Hochschild complex without the term $C^{-1}(A, A)=\operatorname{Hom}_{k}(k, A) \cong A$, while the ordinary cotangent complex would be additionally missing $C^{0}(A, A)$ :

$$
\underbrace{C^{0}(A, A) \stackrel{\bar{\delta}}{\rightarrow} \underbrace{C^{1}(A, A) \stackrel{\bar{\delta}}{\rightarrow} C^{2}(A, A) \stackrel{\bar{\delta}}{\rightarrow} \cdots}_{C^{*}(A, A)}}_{C_{\text {aug }}^{*}(A, A)}
$$

To generalize the conclusion of the example, recall from [14] that $T J$-grading on a free resolution $\mathcal{R}=(\mathbb{F}(X), \partial) \stackrel{\rho}{\rightarrow}(\mathcal{A}, 0)$ is induced by a grading $X=\bigoplus_{i \geqslant 0} X^{i}$ on the $C$-collection of generators, denoted by upper indices, $\mathcal{R}^{i}$, and satisfying

1. $\partial$ maps $X^{i}$ to $\mathbb{F}\left(\bigoplus_{j<i} X^{j}\right)$,

2. $H_{0}\left(\mathcal{R}^{*}, \partial\right) \stackrel{H_{0}(\rho)}{\longrightarrow} \mathcal{A}$ is an isomorphism of graded $C$-operads.

If we have a $T J$-graded resolution $\mathcal{R}$, we can replace the usual grading by the $T J$-grading and we let $\operatorname{Der}_{\mathcal{A}}^{l}\left(\mathcal{R}, \mathcal{E} n d_{A}\right)^{i}$ be the $k$-module of degree $l$ derivations $\mathcal{R} \rightarrow \mathcal{E} n d_{A}$ vanishing on all $X^{j}$ 's except for $j=i$ and ${ }^{T J} C^{*}(A, A):=\left(\operatorname{Der}_{\mathcal{A}}^{1-*}\left(\mathcal{R}, \mathcal{E} n d_{A}\right)^{*-1}, \delta\right)$ and ${ }^{T J} H^{*}(A, A):=H^{*}\left({ }^{T J} C^{*}(A, A)\right)$. In case $\mathcal{A}$ is concentrated in degree 0 , the usual grading is $T J$ and we get the same result as in (11), i.e. $C^{*}(A, A)={ }^{T J} C^{*}(A, A)$ and we can forget about the superscripts $T J$ everywhere.

For a $T J$-graded $\mathcal{R}$, we can also equip $C_{\text {aug }}^{*}(A, A)$ with similar $T J$-grading ${ }^{T J} C_{\text {aug }}^{*}(A, A)$ as above. On this matter we just remark that $\phi$ is placed in $T J$-degree 0 and leave the details for the interested reader. Finally, the following is obvious:

Theorem 3.11 1. ${ }^{T J} C_{\text {aug }}^{n}(A, A)=0$ for $n \leqslant-1$,

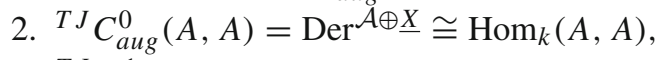

3. ${ }^{T J} H_{\text {aug }}^{1}(A, A) \cong k$-module of formal infinitesimal deformations of the $\mathcal{A}$-algebra structure on A modulo infinitesimal automorphisms,

4. ${ }^{T J} H_{\text {aug }}^{n}(A, A) \cong{ }^{T J} H^{n}(A, A)$ for $n \geqslant 2$. 
Notice that the unaugmented operadic cohomology ${ }^{T J} H^{1}(A, A)$ is the $k$-module of formal infinitesimal deformations of the $\mathcal{A}$-algebra structure on $A$, but the infinitesimal automorphisms are not considered.

Hence the distinction between $H^{*}(A, A)$ and $H_{\text {aug }}^{*}(A, A)$ is inessential and we will usually not distinguish these two.

\section{Intermediate resolution of $\mathcal{D} \mathcal{A}$}

Now we construct an intermediate step in the resolution of Theorem 3.5:

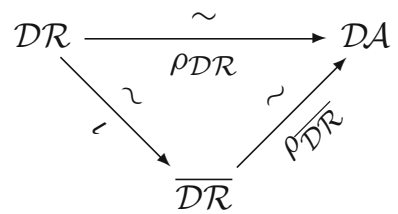

Intuitively, ı should "unresolve" the part of $\mathcal{D} \mathcal{R}$ corresponding to the $\mathcal{A}$-algebra operations and do nothing in the part corresponding to the derivation $\phi$. Let

$$
\overline{\mathcal{D} \mathcal{R}}:=\left(\mathcal{A} * \mathbb{F}(\Phi \oplus \underline{X}), \partial_{\overline{\mathcal{D}}}\right) .
$$

We first define $\iota$ to be the composite

$$
\mathcal{D} \mathcal{R}=\mathbb{F}(X \oplus \Phi \oplus \underline{X}) \cong \mathbb{F}(X) * \mathbb{F}(\Phi \oplus \underline{X}) \stackrel{\rho_{\mathcal{R}} * 1}{\longrightarrow} \mathcal{A} * \mathbb{F}(\Phi \oplus \underline{X})=\overline{\mathcal{D} \mathcal{R}}
$$

then $\partial_{\overline{\mathcal{D}} \mathcal{R}}$ is the derivation defined by

$$
\begin{aligned}
& \partial_{\overline{\mathcal{D R}}}(a):=\partial_{\mathcal{D} \mathcal{A}}(a)=\partial_{\mathcal{A}}(a), \\
& \partial_{\overline{\mathcal{D} \mathcal{R}}}\left(\phi_{c}\right):=0, \\
& \partial_{\overline{\mathcal{D} \mathcal{R}}}(\underline{x}):=\iota\left(\partial_{\mathcal{D} \mathcal{R}} \underline{x}\right) .
\end{aligned}
$$

Now we check $\iota \partial_{\mathcal{D} \mathcal{R}}=\partial_{\overline{\mathcal{D R}}} \iota$ and this will immediately imply $\partial_{\overline{\mathcal{D}} \mathcal{2}}^{2}=0$ :

$$
\begin{aligned}
& \iota \partial_{\mathcal{D} \mathcal{R}}(x)=\iota \partial_{\mathcal{R}}(x)=\rho_{\mathcal{R}} \partial_{\mathcal{R}}(x)=\partial_{\mathcal{A}} \rho_{\mathcal{R}}(x), \\
& \partial_{\overline{\mathcal{D} R}} \iota(x)=\partial_{\overline{\mathcal{D}} \mathcal{R}} \rho_{\mathcal{R}}(x)=\partial_{\mathcal{D} \mathcal{A}} \rho_{\mathcal{R}}(x)=\partial_{\mathcal{A}} \rho_{\mathcal{R}}(x)
\end{aligned}
$$

and similar claim for $\underline{x}$ is an immediate consequence of definitions.

Finally, let $\rho_{\overline{\mathcal{D}} \mathcal{R}}$ be the $C$-operad morphism defined by

$$
\begin{aligned}
& \rho_{\overline{\mathcal{D R}}}(a):=a, \\
& \rho_{\overline{\mathcal{D R}}}\left(\phi_{c}\right):=\phi_{c}, \\
& \rho_{\overline{\mathcal{D R}}}(\underline{x}):=0 .
\end{aligned}
$$


Lemma 4.1 $\rho_{\overline{\mathcal{D} \mathcal{R}}}$ is dg-C-operad morphism.

Proof We only have to check $\rho_{\overline{\mathcal{D} \mathcal{R}}} \partial_{\overline{\mathcal{D} \mathcal{R}}}(\underline{x})=0$ :

$$
\begin{aligned}
\rho_{\overline{\mathcal{D}} \mathcal{R}} \partial_{\overline{\mathcal{D}} \mathcal{R}}(\underline{x}) & =\rho_{\overline{\mathcal{D} \mathcal{R}}} \iota\left(\phi_{c} \circ_{1} x-\sum_{i=0}^{n} x \circ_{i} \phi_{c_{i}}-s\left(\partial_{\mathcal{R}} x\right)\right) \\
& =\phi_{c} \circ_{1} \rho_{\mathcal{R}}(x)-\sum_{i=0}^{n} \rho_{\mathcal{R}}(x) \circ_{i} \phi_{c_{i}} .
\end{aligned}
$$

The third term in the bracket vanishes since $\iota s \partial_{\mathcal{R}}(x)$ is a sum of compositions each of which contains a generator from $\underline{X}$ and $\rho_{\overline{\mathcal{D}} \mathcal{R}}$ vanishes on $\underline{X}$. The above expression vanishes because if $\rho_{\mathcal{R}}(x) \neq 0$, then it is precisely the relator (12).

Lemma 4.2 ı is a quism.

Proof We notice that $\overline{\mathcal{D} \mathcal{R}}$ is close to be the 1 st term of a spectral sequence computing homology of $\mathcal{D} \mathcal{R}$. Now we make this idea precise.

Consider a new grading gr on $\mathcal{D} \mathcal{R}$ :

$$
\operatorname{gr}(x):=0=: \operatorname{gr}(\phi), \quad \operatorname{gr}(\underline{x}):=|\underline{x}|
$$

and its associated filtration

$$
\begin{aligned}
& \mathfrak{F}_{p}:=\bigoplus_{i=0}^{p}\{z \in \mathcal{D} \mathcal{R} \mid \operatorname{gr}(z)=i\}, \\
& 0 \hookrightarrow \mathfrak{F}_{0} \hookrightarrow \mathfrak{F}_{1} \hookrightarrow \cdots, \quad \partial_{\mathcal{D} \mathcal{R} \mathfrak{F}_{p}} \subset \mathfrak{F}_{p}
\end{aligned}
$$

and its associated spectral sequence $\left(E^{*}, \partial^{*}\right)$. There is an analogous spectral sequence $\left(E^{\prime *}, \partial^{* *}\right)$ on $\overline{\mathcal{D} \mathcal{R}}$ given by the grading

$$
\operatorname{gr}^{\prime}(a):=0=: \operatorname{gr}^{\prime}(\phi), \quad \operatorname{gr}^{\prime}(\underline{x}):=|\underline{x}| \text {. }
$$

Since both filtrations are bounded below and exhaustive, we can use the comparison theorem.

We have $E^{0} \cong \mathbb{F}(X \oplus \Phi \oplus \underline{X})$. Recalling the formulas (14), we immediately see that $\partial^{0}$ on $E^{0}$ is the derivation differential given by

$$
\partial^{0} x=\partial_{\mathcal{R}} x, \quad \partial^{0} \phi=0=\partial^{0} \underline{x} .
$$

Hence $E^{1} \cong H_{*}\left(E^{0}, \partial^{0}\right) \cong H_{*}(\mathbb{F}(X)) * H_{*}(\mathbb{F}(\Phi \oplus \underline{X})) \cong \mathcal{A} * \mathbb{F}(\Phi \oplus \underline{X})$ by the Künneth formula for a free product of $\mathrm{dg}$ - $C$-operads, see Lemma 2.4. Similarly $E^{\prime 1} \cong \mathcal{A} * \mathbb{F}(\Phi \oplus \underline{X})$.

Understanding the differentials $\partial^{1}$ and $\partial^{\prime 1}$ on the 1 st pages as well as the induced dg-C-collection morphism $\iota^{1}$ is easy (though notationally difficult - observe $\partial^{1} \underline{x}$ is not $\iota\left(\partial_{\mathcal{D} \mathcal{R}} \underline{x}\right)$ in general!) and we immediately see that $\iota^{1}$ is an isomorphism of $\operatorname{dg}-C$ collections. 
Corollary $4.3 \rho_{\overline{\mathcal{D R}}}$ is a resolution of $\mathcal{D} \mathcal{A}$.

Example 4.4 Let's continue Example 3.6 and make $\overline{\mathcal{D} \mathcal{R}}$ explicit:

$$
\begin{gathered}
\overline{\mathcal{D R}}:=\left(\text { Ass * } \mathbb{F}(\Phi \oplus \underline{X}), \partial_{\overline{\mathcal{D N}}}\right), \\
\partial_{\overline{\mathcal{D R}}}(a):=0=: \partial_{\overline{\mathcal{D R}}}(\phi), \\
\partial_{\overline{\mathcal{D R}}}\left(\underline{x}^{2}\right):=\phi \circ \mu-\mu \circ_{1} \phi-\mu \circ_{2} \phi, \\
\partial_{\overline{\mathcal{D R}}}\left(\underline{x}^{n}\right):=-(-1)^{n} \mu \circ_{2} \underline{x}^{n-1}-\sum_{k=1}^{n-1}(-1)^{n-k} \underline{x}^{n-1} \circ_{k} \mu-\mu \circ_{1} \underline{x}^{n-1}
\end{gathered}
$$

for $n \geqslant 3$. The last formula is reminiscent to the one for the Hochschild differential. We will make this point precise in Sect. 4.1.

\subsection{From operads to operadic modules}

Associated to the operad $\mathcal{D} \mathcal{A}=(\mathcal{A} * \mathbb{F}(\Phi)) / \mathfrak{D}$ is the $\operatorname{dg}-\left(\mathcal{A}, \partial_{\mathcal{A}}\right)$-module

$$
\mathcal{M D A}:=\left(\frac{\mathcal{A}\langle\Phi\rangle}{\mathfrak{D} \cap \mathcal{A}\langle\Phi\rangle}, \partial_{\mathcal{M D A}}\right),
$$

where $\mathfrak{D} \cap \mathcal{A}\langle\Phi\rangle$ is the sub- $\mathcal{A}$-module of $\mathcal{A}\langle\Phi\rangle$ generated by the relators (12) and $\partial_{\mathcal{M D A}}$ is a $\operatorname{dg}-\mathcal{A}$-module morphism given by $\partial_{\mathcal{M D A}} \phi=0$.

Associated to $\overline{\mathcal{D R}}=\left(\mathcal{A} * \mathbb{F}(\Phi \oplus \underline{X}), \partial_{\overline{\mathcal{D R}}}\right)$ is the dg- $\mathcal{A}$-module

$$
\mathcal{M \mathcal { D } \mathcal { R }}:=\left(\mathcal{A}\langle\Phi \oplus \underline{X}\rangle, \partial_{\mathcal{M} \overline{\mathcal{D} R}}\right),
$$

where $\partial_{\mathcal{M}} \overline{\mathcal{D R}}$ is a $\mathcal{A}$-module morphism given by the same formulas (22) as $\partial_{\overline{\mathcal{D R}}}$. We emphasize that this makes sense because $\mathcal{A}\langle\Phi \oplus \underline{X}\rangle \subset \mathcal{A} * \mathbb{F}(\Phi \oplus \underline{X})$, the $\operatorname{dg}-\mathcal{A}$ module structure is induced by the operadic composition and $\partial_{\overline{\mathcal{D R}}}$ maps $\mathcal{A}\langle\Phi \oplus \underline{X}\rangle$ into itself!

Associated to the dg-C-operad morphism $\rho_{\overline{\mathcal{D R}}}: \overline{\mathcal{D R}} \rightarrow \mathcal{D A}$ is the dg- $\mathcal{A}$-module morphism

$$
\rho_{\mathcal{M} \overline{\mathcal{D}}}: \mathcal{M} \overline{\mathcal{D R}} \rightarrow \mathcal{M D A}
$$

again defined by the formulas (23) as $\rho_{\mathcal{M} \overline{\mathcal{D R}}}$.

Lemma $4.5 \rho_{\mathcal{M} \overline{\mathcal{D N}}}$ is a quism.

Proof Let $G_{p}$ be the sub-C-collection of $\overline{\mathcal{D} \mathcal{R}}=\mathcal{A} * \mathbb{F}(\Phi \oplus \underline{X})$ spanned by the compositions containing precisely $p$ generators from $\Phi \oplus \underline{X}$, i.e. $G_{0}=\mathcal{A}, G_{1}=$ 
$\mathcal{A}\langle\Phi \oplus \underline{X}\rangle$ and

$$
\overline{\mathcal{D R}}=\bigoplus_{p \geqslant 0} G_{p} .
$$

We have analogous grading $\mathcal{A} * \mathbb{F}(\Phi)=\bigoplus_{p \geqslant 0} G_{p}^{\prime}$. Let pr : $\mathcal{A} * \mathbb{F}(\Phi) \rightarrow \mathcal{D} \mathcal{A}$ be the natural projection. Since relators (12) are homogeneous with respect to this grading, $G_{p}^{\prime \prime}:=\operatorname{pr} G_{p}^{\prime}$ defines a grading

$$
\mathcal{D} \mathcal{A}=\bigoplus_{p \geqslant 0} G_{p}^{\prime \prime} .
$$

Observe $G_{0}^{\prime \prime}=\mathcal{A}$ and $G_{1}^{\prime \prime}=\mathcal{M D} \mathcal{A}$. By definitions, $\rho_{\overline{\mathcal{D}}} G_{p} \subset G_{p}^{\prime \prime}$, hence $\rho_{\overline{\mathcal{D}} \mathcal{R}}$ decomposes as a sum of $\rho_{\overline{\mathcal{D R}}}{ }^{p}: G_{p} \rightarrow G_{p}^{\prime \prime}$. The above direct sums are in fact direct sums of sub-dg-C-collections, $\rho_{\overline{\mathcal{D R}}}$ is a quism by Corollary 4.3 , hence all the $\rho_{\overline{\mathcal{D R}}}{ }^{\text {'s }}$ are quisms, especially $\rho_{\overline{\mathcal{D} \mathcal{R}}}{ }^{1}=\rho_{\mathcal{M} \overline{\mathcal{D}}}$.

Now we formalize the statement $: \mathcal{M} \overline{\mathcal{D} R}$ contains all the information needed to construct the operadic cohomology for $\mathcal{A}$-algebras.

First observe that $\mathcal{E} n d_{A}$ is naturally a $\operatorname{dg}-\mathcal{A}$-module. Let $\bar{\delta}$ be the differential on $\mathrm{Hom}_{\mathrm{dg}-\mathcal{A}-\bmod }\left(\mathcal{M} \overline{\mathcal{D} \mathcal{R}}, \mathcal{E} n d_{A}\right)$ defined by the formula

$$
\bar{\delta} \theta:=\theta \partial_{\mathcal{M} \overline{\mathcal{D}} \mathcal{R}}-(-1)^{|\theta|} \partial_{\mathcal{E}_{n d_{A}}} \theta
$$

similar to (10).

\section{Lemma 4.6}

$$
\left(C_{\text {aug }}^{*}(A, A), \bar{\delta}\right) \cong\left(\operatorname{Hom}_{\mathrm{dg}-\mathcal{A}-\bmod }\left(\mathcal{M} \overline{\mathcal{D} \mathcal{R}}, \mathcal{E} n d_{A}\right), \bar{\delta}\right)
$$

as dg-k-modules.

Proof On the level of $k$-modules, we have

$$
\begin{aligned}
& C_{\text {aug }}^{*}(A, A)=\operatorname{Der}^{X}=\left\{\theta \in \operatorname{Der}_{\mathcal{A}}\left(\mathbb{F}(X \oplus \Phi \oplus \underline{X}), \mathcal{E}_{n} d_{A}\right) \mid \forall x \in X \quad \theta(x)=0\right\} \\
& \quad \cong \operatorname{Hom}_{\text {dg-C-coll. }}\left(\Phi \oplus \underline{X}, \mathcal{E}_{n d_{A}}\right)
\end{aligned}
$$

by the defining property of derivations and

$$
\operatorname{Hom}_{\mathrm{dg}-\mathcal{A}-\bmod }\left(\mathcal{A}\langle\Phi \oplus \underline{X}\rangle, \mathcal{E} n d_{A}\right) \cong \operatorname{Hom}_{\mathrm{dg}-C-\operatorname{coll} .}\left(\Phi \oplus \underline{X}, \mathcal{E} n d_{A}\right)
$$

by the freeness of $\mathcal{A}\langle\Phi \oplus \underline{X}\rangle$.

The differentials are clearly preserved under the above isomorphism.

The nice thing is that we have now all the information needed to construct the cohomology for $\mathcal{A}$-algebras encoded in terms of the abelian category of $\mathrm{dg}$ - $\mathcal{A}$-modules. Hence 


\section{Theorem 4.7}

$$
\begin{aligned}
H_{\text {aug }}^{*}(A, A) & \cong H_{*}\left(\operatorname{Hom}_{\mathrm{dg}-\mathcal{A}-\bmod }^{-*}\left(\mathcal{M} \overline{\mathcal{D} \mathcal{R}}, \mathcal{E} n d_{A}\right), \bar{\delta}\right) \\
& \cong \mathrm{Ext}_{\mathrm{dg}-\mathcal{A}-\bmod }^{-*}\left(\mathcal{M D} \mathcal{A}, \mathcal{E} n d_{A}\right)
\end{aligned}
$$

In particular, since the homotopy theory in abelian categories is well known and simple, it is immediate that $\operatorname{Ext}_{\mathrm{dg}-\mathcal{A}-\text { mod }}$ and hence $H_{\text {aug }}^{*}(A, A)$ doesn't depend on the choice of a projective resolution of $\mathcal{M D} A$ and consequently doesn't depend on the choice of the free resolution $\mathcal{R} \underset{\rho_{\mathcal{R}}}{\stackrel{\sim}{\longrightarrow}} \mathcal{A}$ in (13).

The main advantage of the above expression is that in order to construct cohomology for $\mathcal{A}$-algebras, we don't need to find a free (or cofibrant) resolution $\mathcal{R} \stackrel{\sim}{\rightarrow} \mathcal{A}$ in the category of $\operatorname{dg}-C$-operads, but it suffices to find a projective resolution of $\mathcal{M D} \mathcal{A}$ in the category of $\mathrm{dg}-\mathcal{A}$-modules, which is certainly easier.

Example 4.8 Let's continue Example 4.4:

$$
\mathcal{M D A}:=\frac{\operatorname{Ass}\langle\Phi\rangle}{\left(\phi \circ \mu-\mu \circ_{1} \phi-\mu \circ_{2} \phi\right)}
$$

and we have the following explicit description of $\mathcal{M} \overline{\mathcal{D R}}$ :

$$
\mathcal{M} \overline{\mathcal{D} \mathcal{R}}:=\left(\mathcal{A} s s\left\langle k\left\langle\phi^{1}, \phi^{2}, \phi^{3}, \ldots\right\rangle\right\rangle, \partial_{\mathcal{M} \overline{\mathcal{D}} \mathcal{R}}\right),
$$

where $\phi^{1}:=\phi$ and $\phi^{n}:=\underline{x}^{n}$, for $n \geqslant 2$, is of degree $n-1$ and the differential is given by

$$
\begin{aligned}
& \partial_{\mathcal{M} \overline{\mathcal{D} R}}\left(\phi^{1}\right):=0, \\
& \partial_{\mathcal{M} \overline{\mathcal{D}}}\left(\phi^{n}\right):=-(-1)^{n} \mu \circ_{2} \phi^{n-1}-\sum_{k=1}^{n-1}(-1)^{n-k} \phi^{n-1} \circ_{k} \mu-\mu \circ_{1} \phi^{n-1} .
\end{aligned}
$$

Lemma 4.5 states that $\mathcal{M} \overline{\mathcal{D R}} \stackrel{\rho_{\mathcal{M} \overline{\mathcal{D R}}}}{\longrightarrow} \mathcal{M D A}$ is a free resolution in the category of Ass-modules.

Notice the similarity to the Hochschild complex. This suggests that if we know a complex computing a cohomology for $\mathcal{A}$-algebras (in this case Hochschild complex) we can read off a candidate for the free resolution of $\mathcal{M D} \mathcal{A}$ (in this case we already know a resolution $\mathcal{M D} \mathcal{A}$, namely $\mathcal{M \mathcal { D } \mathcal { R }}$, but this was constructed from the operadic resolution $\mathcal{R}$, which is not generally available). If we can prove that this candidate is indeed a resolution, we get that the cohomology in question is isomorphic to the augmented operadic cohomology.

We demonstrate this by constructing a cohomology for diagrams of associative algebras and proving that the (augmented) cotangent complex coincides with that defined by Gerstenhaber and Schack [5]. 
On the other hand, in the process of constructing $\mathcal{M} \overline{\mathcal{D} \mathcal{R}}$ we have discarded much information present in $\mathcal{R}$. Namely $\mathcal{R}$ can be used to define an $L_{\infty}$ structure on $C^{*}(A, A)$ governing formal deformations of $A$ (see [13]), which is no longer possible using $\mathcal{M \mathcal { D } R}$ (or any other resolution of $\mathcal{M D A}$ ) only.

\section{Gerstenhaber-Schack diagram cohomology is operadic cohomology}

\subsection{Operad for diagrams}

Let $\mathrm{C}$ be a small category. For a morphism $f$ of $\mathrm{C}$, let $I(f)$ be its source (Input) and $O(f)$ its target (Output). Consider the following nerve construction on C:

$$
\Sigma^{n}:=\left\{\left(\stackrel{f_{n}}{\longleftarrow} \cdots \stackrel{f_{1}}{\longleftarrow}\right) \in \operatorname{Hom}_{\mathrm{C}}^{\times n} \mid O\left(f_{i}\right)=I\left(f_{i+1}\right) \text { for } 1 \leqslant i \leqslant n-1\right\}
$$

for $n \geqslant 1$. For $\sigma=\left(\stackrel{f_{n}}{\longleftarrow} \cdots \stackrel{f_{1}}{\longleftarrow}\right)$, let $|\sigma|:=n$, let $I(\sigma):=I\left(f_{1}\right)$ and $O(\sigma):=$ $O\left(f_{n}\right)$. The face maps $\Sigma^{n} \rightarrow \Sigma^{n+1}$ are given by $\sigma \mapsto \sigma_{i}$, where

$$
\begin{aligned}
& \sigma_{0}:=\left(\stackrel{f_{n}}{\longleftarrow} \cdots \stackrel{f_{2}}{\longleftarrow}\right), \\
& \sigma_{i}:=\left(\stackrel{f_{n}}{\longleftarrow} \cdots \stackrel{f_{i+1} f_{i}}{\longleftarrow} \cdots \stackrel{f_{1}}{\longleftarrow}\right) \text { for } 1 \leqslant i \leqslant n-1, \\
& \sigma_{n}:=\left(\stackrel{f_{n-1}}{\longleftarrow} \ldots \stackrel{f_{1}}{\longleftarrow}\right) .
\end{aligned}
$$

Denote $\Sigma^{0}$ the set of objects of $\mathrm{C}$ and for $\sigma \in \Sigma^{0}$, let $I(\sigma)=O(\sigma)=\sigma$. Finally let

$$
\Sigma:=\bigcup_{n=0}^{\infty} \Sigma^{n}
$$

and denote $\Sigma^{\geqslant 1}:=\Sigma-\Sigma^{0}$.

Let $\mathcal{C}$ be the operadic version of $\mathrm{C}$, that is

$$
\mathcal{C}:=k\left\langle\Sigma^{1}\right\rangle
$$

This can be seen as a $\Sigma^{0}$-operad, where each $f \in \Sigma^{1}$ is an element of $\mathcal{C}\left(\begin{array}{l}O(f) \\ I(f)\end{array}\right)$ and the operadic composition is induced by the categorical composition.

A (C-shaped) diagram (of associative algebras) is a functor

$$
D: \mathrm{C} \rightarrow \mathcal{A} s s \text {-algebras } .
$$


Now we describe a $\Sigma^{0}$-operad $\mathcal{A}$ such that $\mathcal{A}$-algebras are precisely $\mathrm{C}$-shaped diagrams:

$$
\mathcal{A}:=\frac{\left(*_{c \in \Sigma^{0}} \mathcal{A} s s_{c}\right) * \mathcal{C}}{\mathcal{I}},
$$

where $\mathcal{A s s _ { c }}$ is a copy of $\mathcal{A s s}$ concentrated in colour $c$, its generating element is $\mu_{c} \in \mathcal{A} s s_{c}\left(\begin{array}{c}c \\ c, c\end{array}\right)$ and $\mathcal{I}$ is the ideal generated by

$$
f \circ \mu_{I(\sigma)}-\mu_{O(\sigma)} \circ(f, f) \text { for all } f \in \Sigma^{1} \text {. }
$$

It should be clear now that the functor $D$ is essentially the same thing as $\Sigma^{0}$-operad morphism $\mathcal{A} \rightarrow \mathcal{E}_{n d_{A}}$, where $A=\bigoplus_{c \in \Sigma^{0}} D(c)$.

The associated module of Sect. 4.1 is

$$
\mathcal{M D} \mathcal{A}:=\frac{\mathcal{A}\left\langle\bigoplus_{c \in \Sigma^{0}} \Phi_{c}\right\rangle}{\mathfrak{D} \cap \mathcal{A}\left\langle\bigoplus_{c \in \Sigma^{0}} \Phi_{c}\right\rangle}
$$

where $\Phi_{c}=k\left\langle\phi_{c}\right\rangle, \phi_{c}$ being an element of colour $\left(\begin{array}{l}c \\ c\end{array}\right)$ and of degree 0 , and the submodule in the denominator is generated by

$$
\begin{aligned}
& \phi_{c} \circ \mu_{c}-\mu_{c} \circ_{1} \phi_{c}-\mu_{c} \circ_{2} \phi_{c}, \\
& \phi_{O(f)} \circ f-f \circ \phi_{I(f)}
\end{aligned}
$$

for all $c \in \Sigma^{0}$ and all $f \in \mathcal{C}$ (equivalently $f \in \Sigma^{1}$ ). We seek a free resolution $(\mathcal{M R}, \partial) \stackrel{\sim}{\rightarrow}(\mathcal{M D} \mathcal{A}, 0)$ to use Theorem 4.7. Before constructing $\mathcal{M R}$, let's recall the Gerstenhaber-Schack diagram cohomology. As we have seen in Example 4.8, this gives us a candidate for $\mathcal{M} \mathcal{R}$.

\subsection{Gerstenhaber-Schack diagram cohomology}

We adapt the notation from the original source [5]. Originally, the diagram $D$ was restricted to be a poset, but this is unnecessary. Also, instead of associative algebras, one may consider any other type of algebras for which a convenient cohomology is known (e.g. Lie algebras, [6]). In this paper we stick to associative algebras, but we believe that other types can be handled in a similar way.

For $\sigma \in \Sigma^{0}$, denote $\underline{\sigma}:=1_{\sigma}$. For $\sigma=\left(\stackrel{f_{p}}{\longleftarrow} \ldots \stackrel{f_{1}}{\longleftarrow}\right) \in \Sigma^{p}$, denote

$$
\underline{\sigma}:=f_{p} \cdots f_{1}: D(I(\sigma)) \rightarrow D(O(\sigma))
$$

the composition along $\sigma$. This algebra morphisms makes $D(O(\sigma))$ a $D(I(\sigma))$-bimodule. For $p, q \geqslant 0$ let

$$
C_{G S}^{p, q}(D, D):=\prod_{\sigma \in \Sigma^{p}} C_{\mathrm{Hoch}}^{q}(D(I(\sigma)), D(O(\sigma)))
$$


where $C_{\text {Hoch }}^{q}(D(I(\sigma)), D(O(\sigma)))=\operatorname{Hom}_{k}\left(D(I(\sigma))^{\otimes q+1}, D(O(\sigma))\right)$ are the usual Hochschild cochains. We usually abbreviate $C_{G S}^{p, q}:=C_{G S}^{p, q}(D, D)$. There are vertical and horizontal differentials $\delta_{V}: C_{G S}^{p, q} \rightarrow C_{G S}^{p, q+1}$ and $\delta_{H}: C_{G S}^{p, q} \rightarrow C_{G S}^{p+1, q}$. To write them down, let $\sigma=\left(\stackrel{f_{p+1}}{\longleftarrow} \cdots \stackrel{f_{1}}{\longleftarrow}\right) \in \Sigma^{p+1}$ and for $\tau \in \Sigma^{p}$ let $\mathrm{pr}_{\tau}$ : $\prod_{\lambda \in \Sigma^{p}} C_{\text {Hoch }}^{q}(D(I(\lambda)), D(O(\lambda))) \rightarrow C_{\text {Hoch }}^{q}(D(I(\tau)), D(O(\tau)))$ be the projection onto the $\tau$ component of $C_{G S}^{p, q}$. Let $\delta_{\text {Hoch }}$ be the usual Hochschild differential, see (21). Finally, for $\theta \in C_{G S}^{p, q}$, let

$$
\begin{gathered}
\delta_{V}:=(-1)^{p} \prod_{\sigma \in \Sigma^{p}} \delta_{\text {Hoch }}, \\
\operatorname{pr}_{\sigma}\left(\delta_{H} \theta\right):=(-1)^{p+1}\left(\operatorname{pr}_{\sigma_{0}} \theta\right) \circ(\underbrace{f_{1}, \ldots, f_{1}}_{q \text {-times }})+\sum_{i=1}^{p}(-1)^{p+1-i} \operatorname{pr}_{\sigma_{i}} \theta \\
+f_{p+1} \circ\left(\operatorname{pr}_{\sigma_{p+1}} \theta\right) .
\end{gathered}
$$

It is easy to see that $\left(C_{G S}^{*, *}, \delta_{V}, \delta_{H}\right)$ is a bicomplex. The Gerstenhaber-Schack cohomology is defined to be the cohomology of the totalization of this bicomplex,

$$
H_{G S}^{*}(D, D):=H^{*}\left(\bigoplus_{p+q=*} C_{G S}^{p, q}(D, D), \delta_{V}+\delta_{H}\right) .
$$

Notice that we have restricted ourselves to the Hochschild complex without $C_{\text {Hoch }}^{-1}$ as in Example 3.10. Also we consider only the cohomology of $D$ with coefficients in itself as this is the case of interest in the formal deformation theory. The general coefficients can be handled using trivial (operadic) extensions.

\section{Resolution of $\mathcal{M D} \mathcal{A}$}

The preceding section gives us the following candidate for $\mathcal{M R}$ :

$$
\mathcal{M R}:=\left(\mathcal{A}\left\langle\bigoplus_{\sigma \in \Sigma} \Phi_{\sigma} \oplus X_{\sigma}\right\rangle, \partial\right)
$$

where $\Phi_{\sigma}:=k\left\langle\phi_{\sigma}\right\rangle$ with $\phi_{\sigma}$ of colours $\left(\begin{array}{c}O(\sigma) \\ I(\sigma)\end{array}\right)$ and of degree $\left|\phi_{\sigma}\right|:=|\sigma|$ and $X_{\sigma}:=$ $\uparrow|\sigma|+1 X$ is placed in output colour $O(\sigma)$ and input colours $I(\sigma), X$ being the collection of generators of the minimal resolution of Ass as in Example 3.6. The element of $X_{\sigma}$ corresponding to $x \in X$ will be denoted by $x_{\sigma}$, hence $\left|x_{\sigma}^{i}\right|=i-1+|\sigma|$. To define the differential $\partial$ in an economic way, denote $x_{\sigma}^{1}:=\phi_{\sigma}$ for $\sigma \in \Sigma^{0}$ and let

$$
\operatorname{pre}\left(\phi_{\sigma}\right):=0, \operatorname{pre}\left(x_{\sigma}^{i}\right):=x_{\sigma}^{i-1} \text { for } i \geqslant 2
$$


and extend linearly to the generators of $\mathcal{M R}$. Further, let's accept the convention that for $\sigma \in \Sigma^{0}$, the symbol $x_{\sigma_{0}}$ stands for zero. Then

$$
\begin{aligned}
\partial\left(x_{\sigma}\right):= & (-1)^{|\sigma|}\left((-1)^{|x|} \mu_{O(\sigma)} \circ \underline{(\sigma}, \operatorname{pre}\left(x_{\sigma}\right)\right) \\
& \left.+\sum_{i=1}^{\operatorname{ar}(x)-1}(-1)^{|x|-i} \operatorname{pre}\left(x_{\sigma}\right) \circ{ }_{i} \mu_{I(\sigma)}+\mu_{O(\sigma)} \circ\left(\operatorname{pre}\left(x_{\sigma}\right), \underline{\sigma}\right)\right) \\
& +(-1)^{|\sigma|} x_{\sigma_{0}} \circ(\underbrace{f_{1}, \ldots, f_{1}}_{(\operatorname{ar}(x))-\text { times }})+\sum_{i=1}^{|\sigma|-1}(-1)^{|\sigma|-i} x_{\sigma_{i}} f_{|\sigma|} \circ x_{\sigma_{|\sigma|}}
\end{aligned}
$$

for any $\sigma \in \Sigma$. Observe that the first part of the above formula corresponds to the vertical differential (24) and the second part corresponds to the horizontal differential (25) in $C_{G S}^{*}(D, D)$. Then it is easily seen that the dg- $k$-module $\left(\operatorname{Hom}_{\mathrm{dg}-\mathcal{A}-\bmod }(\mathcal{M R}\right.$, $\left.\mathcal{E} n d \bigoplus_{c \in \Sigma^{0}} D(c)\right), \delta$ ) with $\delta(-):=-\circ \partial$ is isomorphic to the Gerstenhaber-Schack complex. Once we prove that $\mathcal{M R}$ is a resolution of $\mathcal{M D} \mathcal{A}$, we will have, by Theorem 4.7 ,

Theorem 6.1 Gerstenhaber-Schack diagram cohomology $H_{G S}^{*}(D, D)$ is isomorphic to the augmented operadic cohomology $H_{\text {aug }}^{*}(D, D)$.

To prove that $\mathcal{M R}$ is a resolution of $\mathcal{M D} \mathcal{A}$ we introduce the $\operatorname{dg}-\mathcal{A}$-module morphism $\rho:(\mathcal{M R}, \partial) \rightarrow(\mathcal{M D} \mathcal{A}, 0)$ given by the formulas

$$
\begin{aligned}
& \rho\left(\phi_{\sigma}\right):=\left\{\begin{array}{lll}
\phi_{\sigma} & \ldots & |\sigma|=0 \\
0 & \ldots & |\sigma| \geqslant 1,
\end{array}\right. \\
& \rho\left(x_{\sigma}\right):=0 .
\end{aligned}
$$

Indeed, it is easy to check that $\rho \partial=0$. It remains to prove

Lemma $6.2 \rho$ is a quism.

Proof is basically a reduction to the following two cases:

1. C is a single object with no morphism except for the identity (Lemma 6.3),

2. C is arbitrary, but each $D(c), c \in \Sigma^{0}$, is the trivial algebra $k$ with zero multiplication (Lemma 6.4).

We first give a general overview of the proof and postpone technicalities to subsequent lemmas.

Consider a new grading on $\mathcal{M R}$ given by

$$
\operatorname{gr}\left(x_{\sigma}\right):=|\sigma|=: \operatorname{gr}\left(\phi_{\sigma}\right)
$$


and the usual requirement that the composition is of degree 0 . Then we have the associated filtration

$$
\begin{aligned}
& \mathfrak{F}_{n}:=\bigoplus_{i=0}^{n}\{x \in \mathcal{M R} \mid \operatorname{gr}(x)=i\}, \\
& 0 \hookrightarrow \mathfrak{F}_{0} \hookrightarrow \mathfrak{F}_{1} \hookrightarrow \cdots, \quad \partial \mathfrak{F}_{i} \subset \mathfrak{F}_{i}
\end{aligned}
$$

and the spectral sequence $\left(E^{*}, \partial^{*}\right)$ which is convergent as the filtration is bounded below and exhaustive.

Obviously $E^{0} \cong \mathcal{M R}$ and $\partial^{0}$ is the derivation differential given by

$$
\begin{aligned}
\partial^{0}\left(x_{\sigma}\right)= & (-1)^{|\sigma|}\left((-1)^{|x|} \mu_{O(\sigma)} \circ\left(\underline{\sigma}, \operatorname{pre}\left(x_{\sigma}\right)\right)\right. \\
& \left.+\sum_{i=1}^{\operatorname{ar}(x)-1}(-1)^{|x|-i} \operatorname{pre}\left(x_{\sigma}\right) \circ_{i} \mu_{I(\sigma)}+\mu_{O(\sigma)} \circ\left(\operatorname{pre}\left(x_{\sigma}\right), \underline{\sigma}\right)\right), \\
\partial^{0}\left(\phi_{\sigma}\right)= & 0 .
\end{aligned}
$$

Now $H_{*}\left(E^{0}, \partial^{0}\right) \cong \bigoplus_{\sigma \in \Sigma} H_{*}\left(\mathcal{A}\left\langle\Phi_{\sigma} \oplus X_{\sigma}\right\rangle, \partial^{0}\right)$ and we use

\section{Lemma 6.3}

$$
H_{*}\left(\mathcal{A}\left\langle\Phi_{\sigma} \oplus X_{\sigma}\right\rangle, \partial^{0}\right) \cong \frac{\mathcal{A}\left\langle\Phi_{\sigma}\right\rangle}{\mathfrak{D}_{\sigma}},
$$

where $\mathfrak{D}_{\sigma}$ is the submodule generated by

$$
\mu_{O(\sigma)} \circ\left(\underline{\sigma}, \phi_{\sigma}\right)+\mu_{O(\sigma)} \circ\left(\phi_{\sigma}, \underline{\sigma}\right)-\phi_{\sigma} \circ \mu_{I(\sigma)} .
$$

This lemma implies

$$
\begin{aligned}
& E^{1} \cong \frac{\mathcal{A}\left\langle\bigoplus_{\sigma \in \Sigma} \Phi_{\sigma}\right\rangle}{\bigoplus_{\sigma \in \Sigma} \mathfrak{D}_{\sigma}}, \\
& \partial^{1}\left(\phi_{\sigma}\right)=(-1)^{|\sigma|} \phi_{\sigma_{0}} \circ f_{1}+\sum_{i=1}^{|\sigma|-1}(-1)^{|\sigma|-i} \phi_{\sigma_{i}}+f_{|\sigma|} \circ \phi_{\sigma_{|\sigma|}} .
\end{aligned}
$$

Denote $\Phi_{\Sigma}:=\bigoplus_{\sigma \in \Sigma} \Phi_{\sigma}$ and write the nominator in the form

$$
\mathcal{A}\left\langle\Phi_{\Sigma}\right\rangle \cong \mathcal{A} \circ^{\prime}\left(I, \Phi_{\Sigma} \circ \mathcal{A}\right)
$$


Here we used the infinitesimal composition product (1). Because of the relations $f \circ \mu_{I(f)}-\mu_{O(f)} \circ(f, f)$ in $\mathcal{A}$ for all $f \in \Sigma^{1}$, we have

$$
\mathcal{A} \cong\left(\bigoplus_{c \in \Sigma^{0}} \mathcal{A} s s_{c}\right) \circ \mathcal{C}
$$

and hence

$$
\mathcal{A}\left\langle\Phi_{\Sigma}\right\rangle \cong \mathcal{A} \circ^{\prime}\left(I, \Phi_{\Sigma} \circ\left(\bigoplus_{c \in \Sigma^{0}} \mathcal{A} s s_{c}\right) \circ \mathcal{C}\right) .
$$

But we are interested in the quotient $E^{1}$ of $\mathcal{A}\left\langle\Phi_{\Sigma}\right\rangle$ and the corresponding relations (27) give us

$$
E^{1} \cong \mathcal{A} \circ^{\prime}\left(I, \Phi_{\Sigma} \circ \mathcal{C}\right)
$$

Now we use (29) again to obtain

$$
E^{1} \cong\left(\bigoplus_{c \in \Sigma^{0}} \mathcal{A} s s_{c}\right) \circ^{\prime}\left(\mathcal{C}, \mathcal{C} \circ \Phi_{\Sigma} \circ \mathcal{C}\right)
$$

Notice that $\mathcal{C} \circ \Phi_{\Sigma} \circ \mathcal{C} \cong \mathcal{C}\left\langle\Phi_{\Sigma}\right\rangle$. Since $\partial^{1}$ is nontrivial only on $\Phi_{\Sigma}$ and $\mathcal{C}\left\langle\Phi_{\Sigma}\right\rangle$ is closed under $\partial^{1}$, to understand $H_{*}\left(E^{1}, \partial^{1}\right)$ using the usual Künneth formula, we only have to compute

\section{Lemma 6.4}

$$
H_{*}\left(\mathcal{C}\left\langle\Phi_{\Sigma}\right\rangle, \partial^{1}\right) \cong \frac{\mathcal{C}\left\langle\bigoplus_{c \in \Sigma^{0}} \Phi_{c}\right\rangle}{\mathfrak{D}^{\prime}},
$$

where $\mathfrak{D}^{\prime}$ is the submodule generated by

$$
f \circ \phi_{I(f)}-\phi_{O(f)} \circ f
$$

for all $f \in \mathcal{C}$.

Then, tracking back all the above isomorphisms, we get

$$
E^{2} \cong H_{*}\left(E^{1}, \partial^{1}\right) \cong \frac{\mathcal{A}\left\langle\bigoplus_{c \in \Sigma^{0}} \Phi_{c}\right\rangle}{\mathfrak{D}^{\prime \prime}},
$$

where $\mathfrak{D}^{\prime \prime}$ is the submodule generated by relators (27) for $\sigma \in \Sigma^{0}$ and all (30)'s. Hence $E^{2} \cong \mathcal{M D A}$ and this is concentrated in degree 0 , the spectral sequence collapses and this concludes the proof of Lemma 6.2. 
Proof of Lemma 6.3 We have already seen in Example 4.8 that for $c \in \Sigma^{0}$ the restriction of $\rho$,

$$
\left(\mathcal{A} s s_{c}\left\langle\Phi_{c} \oplus X_{c}\right\rangle, \partial^{0}\right) \stackrel{\rho}{\rightarrow} \frac{\mathcal{A} s s_{c}\left\langle\Phi_{c}\right\rangle}{\mathfrak{J}_{c}},
$$

is a quism, where $\mathfrak{J}_{c}$ is the submodule generated by (27) for $\sigma=c$. We will reduce our problem to this case. Let

$$
\mathcal{M}_{\sigma}:=\mathcal{A s s}_{O(\sigma)} \circ^{\prime}\left(k\langle\underline{\sigma}\rangle,\left(\Phi_{\sigma} \oplus X_{\sigma}\right) \circ \mathcal{A s s}_{I(\sigma)}\right) .
$$

This is in fact a sub- $\Sigma^{0}$-collection of $\mathcal{A}\left\langle\Phi_{\sigma} \oplus X_{\sigma}\right\rangle$. An easy computation shows that it is closed under $\partial^{0}$. It will play a role similar to $\mathcal{A} s s_{c}\left\langle\Phi_{c} \oplus X_{c}\right\rangle$ above:

Sublemma 6.5 There is an isomorphism

$$
\left(\mathcal{M}_{\sigma}, \partial^{0}\right) \cong \uparrow|\sigma|\left(\mathcal{A s s _ { c }}\left\langle\Phi_{c} \oplus X_{c}\right\rangle, \partial^{0}\right)
$$

of dg-collections (we ignore the colours!). This induces an isomorphism

$$
H_{*}\left(\mathcal{M}_{\sigma}, \partial^{0}\right) \cong \frac{\mathcal{A} s s_{O(\sigma)} \circ^{\prime}\left(k|\underline{\sigma}\rangle, \Phi_{\sigma} \circ \mathcal{A} s s_{I(\sigma)}\right)}{\mathfrak{J}_{\sigma}}
$$

of $\Sigma^{0}$-collections [compare to the right-hand side of (31)], where the quotient by $\mathfrak{J}_{\sigma}$ expresses the fact that $\phi_{\sigma}$ behaves like a derivation with respect to $\mu_{O(\sigma)}$ and $\mu_{I(\sigma)}$ in $\mathcal{M}_{\sigma}$. The relators in $\mathfrak{J}_{\sigma}$ are analogous to (27), namely $\mathfrak{J}_{\sigma}$ is sub- $\Sigma^{0}$-collection of $\mathcal{M}_{\sigma}$ consisting of elements

$$
\begin{aligned}
& \left(a_{O(\sigma)} \circ_{i}\left(\mu_{O(\sigma)} \circ_{1} a_{O(\sigma)}^{1}\right)\right) \circ_{i+\operatorname{ar}\left(a^{1}\right)}^{\prime}\left(\underline{\sigma}, \phi_{\sigma} \circ a_{I(\sigma)}^{2}\right) \\
& +\left(a_{O(\sigma)} \circ_{i}\left(\mu_{O(\sigma)} \circ_{2} a_{O(\sigma)}^{2}\right)\right) \circ_{i}^{\prime}\left(\underline{\sigma}, \phi_{\sigma} \circ a_{I(\sigma)}^{1}\right)+ \\
& -a_{O(\sigma)} \circ_{i}^{\prime}\left(\underline{\sigma}, \phi_{\sigma} \circ \mu_{I(\sigma)} \circ\left(a_{I(\sigma)}^{1}, a_{I(\sigma)}^{2}\right)\right)
\end{aligned}
$$

for all $a, a^{1}, a^{2} \in \mathcal{A}$ ss and $1 \leqslant i \leqslant \operatorname{ar}(a)$.

Proof of Sublemma 6.5 There is a morphism $\psi$ of collections

$$
a_{O(\sigma)} \circ_{i}^{\prime}\left(\underline{\sigma}, x_{\sigma} \circ\left(a_{I(\sigma)}^{1}, \ldots, a_{I(\sigma)}^{\operatorname{ar}(x)}\right)\right) \mapsto a_{c} \circ_{i}^{\prime}\left(1_{c}, x_{c} \circ\left(a_{c}^{1}, \ldots, a_{c}^{\operatorname{ar}(x)}\right)\right)
$$

for $x \in X$ (or $x=\phi$ ) and $a, a^{1}, a^{2}, \ldots \in$ Ass. $\psi$ is obviously an isomorphism of degree $-|\sigma|$. The differential on the suspension is $(-1)^{|\sigma|} \partial^{0}$, hence we must verify

$$
\psi \partial^{0}=(-1)^{|\sigma|} \partial^{0} \psi .
$$

This is immediate by the formula (26) defining $\partial^{0}$. 
Using the relations $f \circ \mu_{I(f)}-\mu_{O(f)} \circ(f, f)$ in $\mathcal{A}$ for $f \in \Sigma^{1}$, every element $a \in \mathcal{A}\left\langle\Phi_{\sigma} \oplus X_{\sigma}\right\rangle$ can be written in the form

$$
a=a_{\text {top }} \circ_{m}\left(a_{\text {mod }} \circ\left(c_{1}, \ldots, c_{\mathrm{ar}\left(a_{\text {mod }}\right)}\right)\right)
$$

for some $a_{\text {top }} \in \mathcal{A}, m \in \mathbb{N}, a_{\text {mod }} \in \mathcal{M}_{\sigma}$ and $c_{1}, c_{2}, \ldots \in \mathcal{C}$. We want to make this is expression as close to being unique as possible. We will require:

If $a_{\text {top }}$ can be written in the form

$$
a_{t o p}=a^{\prime} \circ_{k}\left(\mu_{O(\sigma)} \circ\left(a^{\prime \prime}, 1_{O(\sigma)}\right)\right) \text { or } a_{t o p}=a^{\prime} \circ_{k}\left(\mu_{O(\sigma)} \circ\left(1_{O(\sigma)}, a^{\prime \prime}\right)\right)
$$

for some $a^{\prime}, a^{\prime \prime} \in \mathcal{A}, 1 \leqslant k \leqslant \operatorname{ar}\left(a^{\prime}\right)$ satisfying $m=k+\operatorname{ar}\left(a^{\prime \prime}\right)$ resp. $m=k$, then $\mu_{O(\sigma)} \circ\left(a^{\prime \prime}, a_{m o d}\right)$ resp. $\mu_{O(\sigma)} \circ\left(a_{m o d}, a^{\prime \prime}\right)$ can't be written as an element of $\mathcal{M}_{\sigma} \circ \mathcal{C}$.

It is easily seen that the above requirement can be met for any $a$ so assume it holds in the above expression (32). It is easy to see that this determines $a_{\text {top }}$ and $a_{m o d}$ uniquely up to scalar multiples. The elements $c_{1}, c_{2}, \ldots \in \mathcal{C}$ are however not unique as the following example shows:

Let $x \in X$ and let $f, g_{1}, g_{2} \in \mathcal{C}$ be such that $f g_{1}=f g_{2}$, hence

$$
a:=x_{O(f)} \circ\left(f g_{1}, \phi_{f}\right)=x_{O(f)} \circ\left(f g_{2}, \phi_{f}\right) .
$$

In "the canonical" form (32), $a_{m o d}=x_{O(f)} \circ\left(f, \phi_{f}\right)$, however $c_{1}$ is either $g_{1}$ or $g_{2}$, in pictures:

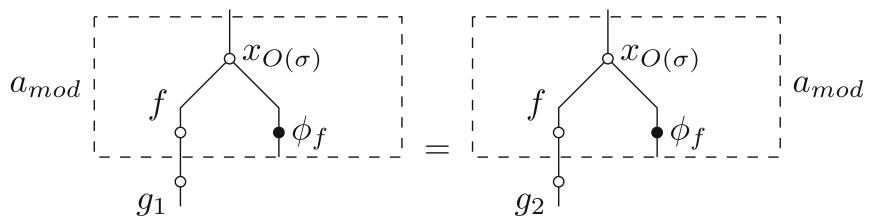

So far we have shown that there are, for $m \geqslant 1, \Sigma^{0}$-collections $\mathcal{A}_{\sigma, m}^{\text {top }}$ (whose description is implicit in the above discussion) such that there is a $\Sigma^{0}$-collection isomorphism

$$
\begin{aligned}
& \mathcal{A}\left\langle\Phi_{\sigma} \oplus X_{\sigma}\right\rangle\left(\begin{array}{c}
c \\
c_{1}, \ldots, c_{N}
\end{array}\right) \\
& \cong \bigoplus_{\substack{m, n \geqslant 1, d \in \Sigma^{0}}} \mathcal{A}_{\sigma, m}^{\text {top }}\left(\begin{array}{c}
c \\
c_{1}, \ldots, c_{m-1}, d, c_{m+n}, \ldots, c_{N}
\end{array}\right) \otimes\left(\frac{\mathcal{M}_{\sigma} \circ \mathcal{C}}{\mathfrak{L}}\right)\left(\begin{array}{c}
d \\
c_{m} \ldots, c_{m+n-1}
\end{array}\right),
\end{aligned}
$$


where $\mathfrak{L}$ is the sub- $\Sigma^{0}$-collection of $\mathcal{M}_{\sigma} \circ \mathcal{C}$ describing the non-uniqueness mentioned above. More precisely, it consists of elements

$$
\underbrace{\left(b \circ_{n}^{\prime}\left(\underline{\sigma,} x \circ\left(b_{1}, \ldots, b_{\operatorname{ar}(x)}\right)\right)\right)}_{a_{\text {mod }}} \circ\left(f_{1}, \ldots, f_{\operatorname{ar}\left(a_{m o d}\right)}\right)
$$

for all $a_{\text {mod }} \in \mathcal{M}_{\sigma}, f_{1}, f_{2}, \ldots \in \mathcal{C}$ satisfying that for some $1 \leqslant i \leqslant n-1$ or $n+\sum_{j=1}^{\operatorname{ar}(x)} \operatorname{ar}\left(b_{j}\right) \leqslant i \leqslant \operatorname{ar}\left(a_{\text {mod }}\right)$ we have $\underline{\sigma} f_{i}=0$. The condition $\underline{\sigma} f_{i}=0$ means that $f_{i}=f-g$ (up to a scalar multiple) for some $f, g \in \Sigma^{1}$ and $\underline{\sigma} f=\underline{\sigma} g$.

At this point we suggest the reader to go through the above discussion in the case $|\sigma|=0$ as many things simplify substantially, e.g. $\mathfrak{L}=0$ and we are essentially done by applying the usual Künneth formula to (33) and then using Sublemma 6.5 which shows

$$
H_{*}\left(\mathcal{M}_{\sigma} \circ \mathcal{C}, \partial^{0}\right) \cong \frac{\left(\mathcal{A} s s_{O(\sigma)} \circ^{\prime}\left(k\langle\underline{\sigma}\rangle, \Phi_{\sigma} \circ \mathcal{A} s s_{I(\sigma)}\right)\right) \circ \mathcal{C}}{\mathfrak{J}_{\sigma}^{\prime}}
$$

where $\mathfrak{J}_{\sigma}^{\prime}$ is an analogue of $\mathfrak{J}_{\sigma}$ above. For general $\sigma$, we have to get rid of the quotient:

\section{Sublemma 6.6}

$$
H_{*}\left(\frac{\mathcal{M}_{\sigma} \circ \mathcal{C}}{\mathfrak{L}}, \partial^{0}\right) \cong \frac{\left(\mathcal{A} s s_{O(\sigma)} \circ^{\prime}\left(I, \Phi_{\sigma} \circ \mathcal{A} s s_{I(\sigma)}\right)\right) \circ \mathcal{C}}{\mathfrak{J}_{\sigma}^{\prime \prime}},
$$

where again $\mathfrak{J}_{\sigma}^{\prime \prime}$ is the corresponding analogue of $\mathfrak{J}_{\sigma}$.

Proof of Sublemma 6.6 Denote

$$
\mathcal{M}_{\sigma} \circ \mathcal{C} \stackrel{\operatorname{pr}}{\rightarrow} \frac{\mathcal{M}_{\sigma} \circ \mathcal{C}}{\mathfrak{L}}=: Q
$$

the natural projection. The differential $\partial_{Q}^{0}$ on $Q$ inherited from $\mathcal{A}\left\langle\Phi_{\sigma} \oplus X_{\sigma}\right\rangle$ is given, for $\alpha \in Q$, by

$$
\partial_{Q}^{0} \alpha=\operatorname{pr} \partial^{0} \tilde{\alpha}
$$

where $\tilde{\alpha} \in \mathcal{M}_{\sigma} \circ \mathcal{C}$ denotes any element such that pr $\tilde{\alpha}=\alpha$.

By (34), to prove the sublemma it suffices to show

1. $\operatorname{pr} \operatorname{Ker} \partial^{0}=\operatorname{Ker} \partial_{Q}^{0}$,

2. $\operatorname{pr} \operatorname{Im} \partial^{0}=\operatorname{Im} \partial_{Q}^{0}$.

For 1., let $\alpha \in Q, \partial_{Q}^{0} \alpha=0$ and we will show there is $\beta \in \mathcal{M}_{\sigma} \circ \mathcal{C}$ satisfying $\partial^{0} \beta=0$ and $\operatorname{pr} \beta=\alpha$. Let $\operatorname{pr} \tilde{\alpha}=\alpha$ and let

$$
\tilde{\alpha}=\sum_{i \in I} a_{m o d}^{i} \circ\left(a_{1}^{i}, \ldots, a_{\operatorname{ar}\left(a_{m o d}^{i}\right)}^{i}\right)
$$


for some index set $I, a_{\text {mod }}^{i} \in \mathcal{M}_{\sigma}$ and $a_{j}^{i} \in \mathcal{C}, i \in I$ and $1 \leqslant j \leqslant \operatorname{ar}\left(a_{\text {mod }}^{i}\right)$, such that any two ordered $\operatorname{ar}\left(a_{m o d}^{i}\right)$-tuples $\left(a_{1}^{i}, \ldots, a_{\operatorname{ar}\left(a_{m o d}^{i}\right)}^{i}\right)$ are distinct for any two distinct $i$ 's. Let $I_{1} \subset I$ be the set of $i$ 's such that $\partial^{0} a_{\text {mod }}^{i}=0$ and let $I_{2}:=I-I_{1}$. By our assumption, $\operatorname{pr} \partial^{0} \tilde{\alpha}=\partial_{Q}^{0} \alpha=0$, hence

$$
\partial^{0} \tilde{\alpha}=\sum_{i \in I_{2}}\left(\partial^{0} a_{\text {mod }}^{i}\right) \circ\left(a_{1}^{i}, \ldots, a_{\operatorname{ar}\left(a_{\text {mod }}^{i}\right)}^{i}\right) \in \mathfrak{L} .
$$

Thus for every $i$ there is $j$ such that $\underline{\sigma} \circ a_{j}^{i}=0$ (by the definition of $\mathfrak{L}$ ). Because $\partial^{0}$ doesn't change $a_{j}^{i}$, s, we get $\sum_{i \in I_{2}} a_{m o d}^{i} \circ\left(a_{1}^{i}, \ldots, a_{\operatorname{ar}\left(a_{m o d}^{i}\right)}^{i}\right) \in \mathfrak{L}$ and we set

$$
\beta:=\sum_{i \in I_{1}} a_{m o d}^{i} \circ\left(a_{1}^{i}, \ldots, a_{\operatorname{ar}\left(a_{m o d}^{i}\right)}^{i}\right)
$$

Then obviously $\partial^{0} \beta=0$ and $\alpha=\operatorname{pr} \beta$, so we have obtained $\operatorname{pr} \operatorname{Ker} \partial^{0} \supset \operatorname{Ker} \partial_{Q}^{0}$. The opposite inclusion is obvious. Also 2. is easy.

Now apply Sublemma 6.6 to (33). This concludes the proof of Lemma 6.3.

Proof of Lemma 6.4 This is just a straightforward application of Lemma 4.5 to operad $\mathcal{A}:=\mathcal{C}$ and its bar-cobar resolution $\mathcal{R}:=\Omega B \mathcal{C}$ (e.g. [19]). To see this, recall that $\Omega \mathrm{BC}$ is a quasi-free $\Sigma^{0}$-operad generated by $\Sigma^{0}$-collection $k\left\langle\Sigma^{\geqslant 1}\right\rangle$, where the degree of $\sigma \in \Sigma \geqslant 1$ is $|\sigma|-1$. The derivation differential is given by

$$
\begin{aligned}
& \partial \underbrace{\left(f_{n} \ldots \stackrel{f_{1}}{\longleftarrow}\right)}_{\sigma}:=\sum_{i=1}^{n-1}(-1)^{i+n+1}\left(\stackrel{f_{n}}{\longleftarrow} \ldots \stackrel{f_{i+1}}{\longleftarrow}\right) \circ\left(\stackrel{f_{i}}{\longleftarrow} \ldots \stackrel{f_{1}}{\longleftarrow}\right) \\
& +\sum_{i=1}^{n-1}(-1)^{n-i} \underbrace{\left(\stackrel{f_{n}}{\longleftarrow} \cdots f_{i+1} f_{i}\right.}_{\sigma_{i}} \cdots \stackrel{f_{1}}{\longleftarrow}) .
\end{aligned}
$$

The projection $\Omega \mathrm{BC} \stackrel{\rho_{\mathcal{R}}}{\longrightarrow} \Omega \mathrm{B}^{1} \mathcal{C} \cong \mathcal{C}$ onto the sub- $\Sigma^{0}$-collection consisting of single generators is a quism.

Then $\mathcal{M} \overline{\mathcal{D R}}$ of Lemma 4.5 is

$$
\mathcal{M} \overline{\mathcal{D} \Omega \mathrm{BC}}=\left(\mathcal{A}\left\langle\bigoplus_{c \in \Sigma^{0}} \Phi_{c} \oplus \bigoplus_{\sigma \in \Sigma \geqslant 1} \Phi_{\sigma}\right\rangle, \partial_{\mathcal{M}} \overline{\mathcal{D} \Omega \mathrm{BC}}\right)
$$

where all the symbols have the same meaning as in the previous parts of this paper and it is easily checked that the differential is given by the formula (28). This resolves $\mathcal{M D C}$, which is readily seen to be the right-hand side in the statement of Lemma 6.4. 


\section{References}

1. Fox, T.F., Markl, M.: Distributive laws, bialgebras, and cohomology. Contemp. Math., 202, Am. Math. Soc., Providence, RI (1997)

2. Frégier, Y., Markl, M., Yau, D.: The L1-deformation complex of diagrams of algebras. NY. J. Math. 15, 353-392 (2009)

3. Fresse, B.: Modules Over Operads and Functors. In: Lecture Notes in Mathematics, 1967, Springer, Berlin (2009) ISBN:978-3-540-89055-3

4. Galvez-Carrillo, I., Tonks, A., Vallette, B.: Homotopy Batalin-Vilkovisky algebras. arXiv (2010)

5. Gerstenhaber, M., Schack S.D.: On the deformation of algebra morphisms and diagrams. Trans. Am. Math. Soc. 279/1, 1-50 (1983)

6. Gerstenhaber, M., Giaquinto, A., Schack S.D.: Diagrams of lie algebras. J. Pure Appl. Algebra 196, 169-184 (2005)

7. Ginzburg, V., Kapranov M.: Koszul duality for operads. Duke Math. J. 76(1), 203-272 (1994)

8. Loday, J.-L., Vallette, B.: Algebraic operads. (available at the web pages of the authors)

9. Markl, M.: A resolution (minimal model) of the PROP for bialgebras, J. Pure Appl. Algebra 205(2), 341-374 (2006)

10. Markl, M.: Cotangent cohomology of a category and deformations. J. Pure Appl. Algebra 113(2), 195-218 (1996)

11. Markl, M.: Homotopy algebras are homotopy algebras. Forum Math. 16(1), 129-160 (2004)

12. Markl, M.: Homotopy diagrams of algebras. In: Proceedings of the 21 st Winter School Geometry and Physics (Srní, 2001), Rend. Circ. Mat. Palermo (2) Suppl. 2002, no. 69, 161-180

13. Mark1, M.: Intrinsic brackets and the L1-deformation theory of bialgebras. J. Homotopy Relat. Struct. 5(1), 177-212 (2010)

14. Markl, M.: Models for operads. Comm. Alg. 24, 1471-1500 (1996)

15. Markl, M., Remm, E.: (Non-)Koszulness of operads for n-ary algebras, galgalim and other curiosities. $\operatorname{arXiv}(2009)$

16. Markl, M., Shnider, S., Stasheff, J.: Operads in algebra, topology and physics. Mathematical Surveys and Monographs, 96, AMS, Providence, RI (2002). ISBN:0-8218-2134-2

17. Merkulov, S., Vallette, B.: Deformation theory of representations of prop(erad)s I. J. Reine Angew. Math. 634, 51-106 (2009)

18. Merkulov, S., Vallette, B.: Deformation theory of representations of prop(erad)s II. J. Reine Angew. Math. 636, 123-174 (2009)

19. Vallette, B.: A Koszul duality for props. Trans. Am. Math. Soc. 359, 4865-4943 (2007)

20. Weibel, C.A.: An introduction to homological algebra. Cambridge Studies in Advanced Mathematics, 38, Cambridge University Press, Cambridge (1994). ISBN:0-521-43500-5; 0-521-55987-1 